\title{
A class of three-dimensional Ricci solitons
}

\author{
PAUL BAIRD
}

\begin{abstract}
We describe a three-dimensional autonomous dynamical system, orbits of which determine the metrics of three-dimensional Ricci solitons. In general these are not of gradient type. A careful analysis of the asymptotic behaviour of orbits is required to establish whether the corresponding solitons are complete or otherwise. New examples are found. Special cases include soliton structures on surfaces. In particular, a non-gradient steady soliton is found on an infinite cover of $S^{2} \backslash\{$ two points $\}$ whose metric factors then extends to a non-standard $C^{2}$ metric on $S^{2}$.
\end{abstract}

57M50, 35Q51

\section{Introduction}

Let $\left(M, g_{0}\right)$ be a Riemannian manifold. Then the Ricci flow is the evolution equation:

$$
\left\{\begin{array}{l}
\frac{\partial g}{\partial t}=-2 \operatorname{Ricci}(g) \\
g(0)=g_{0} .
\end{array}\right.
$$

Considerable advances were made in understanding the behaviour of solutions, with the introduction by Perelman of the functional (called Perelman's entropy):

$$
\mathcal{W}(g, f, \tau)=\int_{M}(4 \pi \tau)^{-n / 2} e^{-f}\left(\tau\left(|\operatorname{grad} f|^{2}+R\right)+f-n\right) \mathrm{d} v_{g}
$$

where $R$ is the scalar curvature, $\tau$ is a positive scalar function of $t$ and $f$ is a smooth function subject to the constraint that the measure $d \mu:=(4 \pi \tau)^{-n / 2} e^{-f} \mathrm{~d} v_{g}$ remains constant. The functional is invariant under simultaneous scalings of $\tau$ and $g$ and under diffeomorphism: $\mathcal{W}(g, f, \tau)=\mathcal{W}\left(c \psi^{*} g, \psi^{*} f, c \tau\right)$ and the corresponding gradient flow is equivalent to (1) up to diffeomorphism (Perelman [11]). The monotonicity formula:

$$
\frac{\mathrm{d}}{\mathrm{d} t} \mathcal{W}=\int_{M} 2 \tau\left|\operatorname{Ricci}+\nabla^{2} f-\frac{1}{2 \tau} g\right|^{2}(4 \pi \tau)^{-n / 2} e^{-f} d v_{g}
$$


shows that a critical metric corresponds to a (gradient) Ricci soliton, that is a metric satisfying the equation (cf Chow et al [3, Proposition 1.7]):

$$
\operatorname{Ricci}(g)+\nabla^{2} f+A g=0,
$$

where $A$ is constant, and in favourable circumstances, we may expect convergence in some appropriate limit to such a metric up to diffeomorphism.

More generally, a Ricci soliton is a metric $g$ satisfying the equation:

$$
-2 \operatorname{Ricci}(g)=\mathcal{L}_{E} g+2 A g,
$$

where $E$ is a vector field called the soliton flow, $\mathcal{L}_{E}$ denotes Lie derivation with respect to $E$ and $A$ is constant. The soliton is called shrinking, steady or expanding when $A<0,=0,>0$, respectively. In the case when $E=\operatorname{grad} f$ is the gradient of a function, then $\mathcal{L}_{E} g=2 \nabla \mathrm{d} f$ and the soliton is said to be of gradient type. Any solution to (1) of the form $g(t)=c(t) \psi_{t}^{*}(g)$, where $c(t)$ is a scalar function and $\left\{\psi_{t}\right\}$ is a smoothly varying family of diffeomorphisms satisfying $\psi_{0}=\mathrm{id}$, has the property that $g(0)$ satisfies (5) with $c^{\prime}(0)=2 A$ and $E(x)=\left.\frac{\mathrm{d}}{\mathrm{d} t} \psi_{t}(x)\right|_{t=0}$ for all $x \in M$. Conversely, any complete metric satisfying (5) determines a solution of (1) of this type for small time (see Chow-Knopf [4]). So a soliton corresponds to a solution of the Ricci flow evolving by diffeomorphism and scaling.

Non-gradient solitons play a fundamental role in the study of the long-term behaviour of the Ricci flow and until recently no examples were known. In Baird-Danielo [1], a complete description of the soliton structures on the eight three-dimensional geometries of Thurston was obtained, in particular non-gradient structures were found on the geometries Nil and Sol, while it was shown that the geometry $\widetilde{S L_{2}(\mathbf{R})}$ carries no soliton structure whatsoever. Independently, J Lott found the same soliton structures as well as similar ones on the four-dimensional geometries [10]. Furthermore, he showed that on a class of manifolds, if $g(t)$ is a solution to (1), then the sequence of Ricci flows: $\left\{g_{s}(t)=s^{-1} g(s t)\right\}$, which exhibit the long time behaviour of $g(t)$, converge to a soliton flow in the sense of Hamilton's pointed convergence [8]. Otherwise, Guenther, Isenberg and Knopf have used the non-gradient soliton structures on Nil and Sol to study the stability of a solution in a neighbourhood of metrics about a metric induced from one of these geometries Guenther-Isenberg-Knopf [6; 7]. In the Ricci flat case, the stability is also studied by N Sesum [12].

In spite of their importance, it is therefore surprising that few explicit examples of 3dimensional solitons are known. Any metric of constant curvature satisfies Ricci $(g)=$ $c g$ for a constant $c$ and is therefore a soliton: we take $E$ to be zero or any Killing field $\left(\mathcal{L}_{E} g=0\right)$. By a result of Ivey, any 3-dimensional soliton of non-constant curvature 
is non-compact [9]. The rotationally symmetric Gaussian soliton on $\mathbf{R}^{n}$ is of gradient type with $E=\operatorname{grad} a|x|^{2}$ for some constant $a$; it shows that a manifold may carry nonunique soliton structures. It is easily seen that the cylinders $S^{2} \times \mathbf{R}$ and $H^{2} \times \mathbf{R}$ admit unique (gradient) soliton structures, where $S^{2}$ and $H^{2}$ are standard 2-dimensional spherical and hyperbolic space, respectively, whereas Nil and Sol admit unique (nongradient) soliton structures (Baird-Danielo[1], Lott [10]). Together with the fact that the geometry $\widetilde{S L_{2}(\mathbf{R})}$ carries no soliton structure, this gives a complete description on the 3-dimensional geometries. Another example is the rotationally symmetric gradient stationary soliton of Bryant, whose metric has the form $g=\mathrm{d} r^{2}+a(r)^{2} g_{S^{2}}$, where $g_{S^{2}}$ is the standard metric on the 2 -sphere and where $a(r)$ is derived from a 2-dimensional autonomous dynamical system (Bryant [2], Chow et al [3]). Threedimensional solitons also derive from a soliton structure on a surface $\Sigma$ by taking the product $\Sigma \times \mathbf{R}$. A fundamental example is when $\Sigma$ is Hamilton's 2-dimensional cigar; this is a rotationally symmetric 2 -dimensional gradient soliton and is the only complete stationary gradient soliton with positive curvature in dimension 2 [8].

Our aim in this paper is to construct new examples from a dynamical system. We note that in coordinates $\left(x^{1}, x^{2}, x^{3}\right)$, many of the metrics $g=g_{i j} \mathrm{~d} x^{i} \mathrm{~d} x^{j}$ in the above list of examples can be expressed in the form:

$$
\left(g_{i j}\right)=\left(\begin{array}{ccc}
1 & 0 & 0 \\
0 & a^{2}+e^{2} & e b \\
0 & e b & b^{2}
\end{array}\right)
$$

where the three functions $a, b, e$ all depend on $x^{1}$ only. It is important to note however, that the vector field $E$ defining the soliton flow will generally depend on all three variables $\left(x^{1}, x^{2}, x^{3}\right)$. In the next section, we show how such metrics arise naturally under the hypothesis that the soliton $\left(M^{3}, g\right)$ admits a semi-conformal mapping onto a surface. On writing $t=x^{1}$, the soliton equations become an autonomous dynamical system in $\mathbf{R}^{5}$ (Theorem 3.3 - with $t$ now taken to be the time coordinate of a solution curve, not to be confused with the time evolution parameter in the Ricci flow (1)). Each orbit determines a soliton metric, but in general it is difficult to extract useful information about its nature. However, when we restrict to metrics such that the horizontal distribution of the corresponding semi-conformal mapping is integrable (up to diffeomorphism $e \equiv 0$ in (6)), we now obtain an autonomous dynamical system in $\mathbf{R}^{3}$ which becomes more amenable to study (Theorem 3.1).

The system depends on two parameters $k$ and $A$, the latter, which corresponds to the constant in (5), can be normalised to have the values $-2,0$, or 2 . But now, different values of the first parameter $k$ yield different soliton structures in general, for example, apart from exceptional cases, the soliton is of gradient type if and only if $k+A=0$. 
Fixed points correspond to the geometry Sol and all the other geometries apart from Nil and $\widetilde{S L_{2}(\mathbf{R})}$ (which doesn't admit any soliton structure at all) are recognizable as orbits.

We undertake a phase space analysis of the dynamical system, and in particular show that there exists an invariant 2-dimensional subspace (Lemma 4.1). Restriction to this subspace now determines a 2-dimensional autonomous system. In general, orbits approach infinity in a finite time and a careful analysis of the asymptotic behaviour of these orbits is required to see whether the corresponding metrics extend to complete ones. When $A \neq 0$, the new examples we construct are not complete, however, when $A=0$ and $k \neq 0$, we find an interesting family of examples, equivalent by homothety, which have the form of a product $\Sigma \times \mathbf{R}$, where $\Sigma$ is a 2 -dimensional non-gradient soliton diffeomorphic to $\mathbf{R}^{2}$. Although the soliton flow doesn't factor, the metric factors to the cylinder $\mathbf{R} \times S^{1}$ and by the cylinder to ball rule, extends to a non-standard $C^{2}$ metric $g_{0}$ on the $2-$ sphere $S^{2}$. As such, we can view the soliton structure as living on an infinite branched cover of $S^{2} \backslash$ \{two points\}. Furthermore the evolution of $\left(S^{2}, g_{0}\right)$ under the Ricci flow is governed by the soliton evolution by diffeomorphism on $\Sigma$. The key elements of this example are summarized in Theorem 5.12 and the preceeding discussion.

\section{Normalisation of the metric}

A smooth map $\phi:\left(M^{m}, g\right) \rightarrow\left(N^{n}, h\right)$ between manifolds $M, N$ with respective Riemannian metrics $g, h$ is said to be semi-conformal if, for each $x \in M$ where $\mathrm{d} \phi_{x} \neq 0$, the restriction $\left.\mathrm{d} \phi_{x}\right|_{\mathcal{H}_{x}}: \mathcal{H}_{x} \rightarrow T_{\phi(x)} N$ is conformal and surjective, where $\mathcal{H}_{x}=\left(\operatorname{ker} \mathrm{d} \phi_{x}\right)^{\perp}$. Thus there exists a number $\lambda=\lambda(x)>0$ such that $\phi^{*} h(X, Y)=$ $\lambda(x)^{2} g(X, Y)$ for all $X, Y \in \mathcal{H}_{x}$. Setting $\lambda=0$ at points $x \in M$ where $\mathrm{d} \phi_{x}=0$, we obtain a continuous function $\lambda: M \rightarrow \mathbf{R}(\geq 0)$ called the dilation of $\phi$; it has the property that $\lambda^{2}=\frac{1}{n}\|\mathrm{~d} \phi\|^{2}$ is smooth, where $\|\mathrm{d} \phi\|$ represents the Hilbert-Schmidt norm of the derivative at each point. If we now suppose that $\operatorname{dim} M=\operatorname{dim} N+1$ with both $M$ and $N$ oriented (or simply, the fibres of $\phi$ are oriented) and that $\phi$ is submersive, then it follows that the metric $g$ has the form

$$
g=\frac{\phi^{*} h}{\lambda^{2}}+\theta^{2}
$$

for some 1 -form $\theta$ on $M$, non-zero on $\operatorname{ker} \mathrm{d} \phi$.

Now suppose $\operatorname{dim} M=3$ and $\operatorname{dim} N=2$ and choose local coordinates with respect to which $\phi$ is the projection $\phi\left(x^{1}, x^{2}, x^{3}\right)=\left(x^{1}, x^{2}\right)$. Then $g=g_{i j} \mathrm{~d} x^{i} \mathrm{~d} x^{j}$ has the 
form

$$
\left(g_{i j}\right)=\left(\begin{array}{ccc}
\frac{h_{11}}{\lambda^{2}}+f_{1}^{2} & \frac{h_{12}}{\lambda^{2}}+f_{1} f_{2} & f_{1} b \\
\frac{h_{12}}{\lambda^{2}}+f_{1} f_{2} & \frac{h_{22}}{\lambda^{2}}+f_{2}^{2} & f_{2} b \\
f_{1} b & f_{2} b & b^{2}
\end{array}\right)
$$

where we have written $\theta=f_{1} \mathrm{~d} x^{1}+f_{2} \mathrm{~d} x^{2}+b \mathrm{~d} x^{3}$, with $b$ a function everywhere non-zero and where the functions $h_{i j}$ depend on $\left(x^{1}, x^{2}\right)$ only. We will make the further assumptions that the coordinates $\left(x^{1}, x^{2}\right)$ on $N$ are geodesic, so that $h=$ $\left(\mathrm{d} x^{1}\right)^{2}+a\left(x^{1}\right)^{2}\left(\mathrm{~d} x^{2}\right)^{2}$, that the functions $f_{1}, f_{2}, b$ depend on $x^{1}$ only and that $\lambda \equiv 1$. We shall write $t=x^{1}$, so we now have the form:

$$
\left(g_{i j}\right)=\left(\begin{array}{ccc}
1+f_{1}^{2} & f_{1} f_{2} & f_{1} b \\
f_{1} f_{2} & a^{2}+f_{2}^{2} & f_{2} b \\
f_{1} b & f_{2} b & b^{2}
\end{array}\right)
$$

where all functions $a, b, f_{1}, f_{2}$ depend on $t$ only. As already remarked, when the metric is that of a soliton, it is usually the case that the soliton flow given by the vector field $E$ in (5) depends on all three variables $\left(x^{1}, x^{2}, x^{3}\right)$. The change of variable: $\widetilde{x^{3}}=x^{3}+\gamma\left(x^{1}\right)$, where $\gamma\left(x^{1}\right)=\int^{x^{1}} \frac{f_{1}(t)}{b(t)} \mathrm{d} t$, transforms the metric (7) to give

$$
g\left(x^{1}, x^{2}, \widetilde{x^{3}}\right)=\left(\mathrm{d} x^{1}\right)^{2}+a^{2}\left(\mathrm{~d} x^{2}\right)^{2}+\left(f_{2} \mathrm{~d} x^{2}+b \mathrm{~d} \widetilde{x^{3}}\right)^{2}
$$

so that, under diffeomorphism, it is no loss of generality to suppose $f_{1} \equiv 0$ and on writing $e$ for the function $f_{2}$, we recover the normalised form of the introduction:

$$
\left(g_{i j}\right)=\left(\begin{array}{ccc}
1 & 0 & 0 \\
0 & a^{2}+e^{2} & e b \\
0 & e b & b^{2}
\end{array}\right) .
$$

A further normalisation can be achieved in the case when the horizontal space $\mathcal{H}$ (the compliment of ker $\mathrm{d} \phi$ ) is integrable. A horizontal frame is given by $\left\{Y_{1}, Y_{2}\right\}$ where $Y_{1}=\frac{\partial}{\partial x^{1}}$ and $Y_{2}=\frac{\partial}{\partial x^{2}}-\frac{e}{b} \frac{\partial}{\partial x^{3}}$. Then the Lie bracket $\left[Y_{1}, Y_{2}\right]=-\left(\frac{e}{b}\right)^{\prime} \frac{\partial}{\partial x^{3}}$, which vanishes if and only if $\frac{e}{b}=$ const. If this constant is non zero, we define new coordinates by setting

$$
\left\{\begin{array}{l}
\widetilde{x^{2}}=\left(\frac{e}{b}\right) x^{2} \\
\widetilde{x^{3}}=\left(\frac{e}{b}\right) x^{2}+x^{3}
\end{array}\right.
$$

Clearly the transformation is a diffeomorphism (being invertible) and with respect to the new coordinates, the metric $g$ is diagonal of the form:

$$
g\left(x^{1}, \widetilde{x^{2}}, \widetilde{x^{3}}\right)=\left(\mathrm{d} x^{1}\right)^{2}+a^{2} \frac{b^{2}}{e^{2}}\left(\widetilde{\mathrm{d}} \widetilde{x^{2}}\right)^{2}+b^{2}\left(\widetilde{\mathrm{d} x^{3}}\right)^{2} .
$$


We shall therefore also distinguish the normalised form:

$$
\left(g_{i j}\right)=\left(\begin{array}{ccc}
1 & 0 & 0 \\
0 & a^{2} & 0 \\
0 & 0 & b^{2}
\end{array}\right)
$$

in the case when $\mathcal{H}$ is integrable.

It is useful to have expressions for the Christoffel symbols

$$
\Gamma_{i j}^{k}=\frac{1}{2} g^{k l}\left(\partial_{i} g_{j l}+\partial_{j} g_{i l}-\partial_{l} g_{i j}\right)
$$

where $\left(g^{k l}\right)$ is the inverse matrix $\left(g_{i j}\right)^{-1}$ and $\partial_{i}=\partial / \partial x^{i}$, etc, and the components of the Ricci curvature:

$$
R_{j k}=\partial_{l} \Gamma_{j k}^{l}-\partial_{j} \Gamma_{l k}^{l}+\Gamma_{l m}^{l} \Gamma_{j k}^{m}-\Gamma_{j m}^{l} \Gamma_{l k}^{m}
$$

Although it is straightforward to calculate these for the form (8), we will only require them in the diagonal case (9). It will be useful to write $u=b^{\prime} / b$ and $v=a^{\prime} / a$ in what follows.

\section{Lemma 2.1}

(i) The Christoffel symbols of the metric (9) are given as follows: all symbols without the index 1 vanish; for those containing the index 1, we have:

$$
\begin{aligned}
\Gamma_{11}^{1}= & \Gamma_{12}^{1}=\Gamma_{13}^{1}=\Gamma_{11}^{2}=\Gamma_{11}^{3}=\Gamma_{23}^{1}=\Gamma_{13}^{2}=\Gamma_{12}^{3}=0 \\
& \Gamma_{22}^{1}=-a a^{\prime}, \quad \Gamma_{33}^{1}=-b b^{\prime}, \quad \Gamma_{12}^{2}=v, \quad \Gamma_{13}^{3}=u .
\end{aligned}
$$

(ii) The Ricci curvature of the metric (9) is diagonal with components:

$R_{11}=-\left(u^{\prime}+v^{\prime}+u^{2}+v^{2}\right), \quad R_{22}=-a^{2}\left(v^{\prime}+u v+v^{2}\right), \quad R_{33}=-b^{2}\left(u^{\prime}+u^{2}+u v\right)$.

Corollary 2.2 The mean curvature of the fibres of $\phi$ is given by $\mu=-u \partial_{1}$, in particular, a potential for the vector field $\mu$ is given by the function $\ln b^{-1}$.

Proof This is a consequence of the definition: $\mu=\frac{1}{b^{2}} \nabla_{\partial_{3}} \partial_{3}=\frac{1}{b^{2}} \Gamma_{33}^{k} \partial_{k}$.

Corollary 2.3 The metric (9) has constant curvature if and only if

$$
\left\{\begin{array}{l}
\text { (i) } \quad u^{\prime}+u^{2}-u v=0 \\
\text { (ii) } \quad v^{\prime}+v^{2}-u v=0
\end{array}\right.
$$

with $u v=$ const. Note that if $u v$ is a non-zero constant then (i) and (ii) are equivalent. 
Proof The metric has constant curvature if and only if Ricci $(g)=-\lambda g$ for some constant $\lambda$. This condition is equivalent to the three equations:

$$
\left\{\begin{aligned}
u^{\prime}+v^{\prime}+u^{2}+v^{2} & =\lambda \\
v^{\prime}+v^{2}+u v & =\lambda \\
u^{\prime}+u^{2}+u v & =\lambda .
\end{aligned}\right.
$$

Summing the last two of these and applying the first gives $\lambda=2 u v$. Substituting back, we find that the first equation is a consequence of the second and third, which now have the form stated in the corollary.

To finish this section and for future reference, we list the eight geometries together with their corresponding metrics in normalized form:

$$
\begin{array}{clc}
\mathbf{R}^{3}:\left(\begin{array}{lll}
1 & 0 & 0 \\
0 & 1 & 0 \\
0 & 0 & 1
\end{array}\right) & S^{3}:\left(\begin{array}{ccc}
1 & 0 & 0 \\
0 & \sin ^{2} t & 0 \\
0 & 0 & \cos ^{2} t
\end{array}\right) \\
H^{3}:\left(\begin{array}{ccc}
1 & 0 & 0 \\
0 & \sinh ^{2} t & 0 \\
0 & 0 & \cosh ^{2} t
\end{array}\right) & S^{2} \times \mathbf{R}:\left(\begin{array}{ccc}
1 & 0 & 0 \\
0 & \sin ^{2} t & 0 \\
0 & 0 & 1
\end{array}\right) \\
H^{2} \times \mathbf{R}:\left(\begin{array}{ccc}
1 & 0 & 0 \\
0 & \sinh ^{2} t & 0 \\
0 & 0 & 1
\end{array}\right) & \text { Nil : } & \left(\begin{array}{ccc}
1 & 0 & 0 \\
0 & 1+t^{2} & t \\
0 & t & 1
\end{array}\right) \\
\widetilde{S L_{2}(\mathbf{R}):}\left(\begin{array}{ccc}
1 & 0 & 0 \\
0 & 2 e^{-2 t} & e^{-t} \\
0 & e^{-t} & 1
\end{array}\right) & \text { Sol : }\left(\begin{array}{ccc}
1 & 0 & 0 \\
0 & e^{2 t} & 0 \\
0 & 0 & e^{-2 t}
\end{array}\right) .
\end{array}
$$

\section{The dynamical system}

We derive a 3-dimensional autonomous dynamical system whose orbits correspond to soliton metrics in diagonal form (9). For the more general case (8), a 5-dimensional dynamical system can be deduced from the ansatz derived in [1]. This contains the 3-dimensional system as an invariant subspace. As all our examples come from the 3dimensional system, we will prove that case and just state the form of the 5-dimensional one and make some general observations in that case.

First we decompose the vector field $E$ in (5) into horizontal and vertical components:

$$
E=X+f U
$$

where $U=\frac{1}{b} \partial_{3}$ is the unit vertical vector field and $f$ is some function. We suppose further that $X=\operatorname{grad} \beta \circ \phi$. This is not the same as supposing that $E$ is of gradient 
type, but rather that its horizontal component is. One of the useful consequences in assuming this decomposition in $E$ is to essentially fix a gauge for $E$, since in general, the soliton flow is defined only up to addition of a Killing vector field. We will now find that orbits of our dynamical system are independent of any freedom that remains in the choice of $E$.

Theorem 3.1 Let $A$ and $k$ be constants and consider the 3-dimensional autonomous dynamical system:

$$
\left\{\begin{array}{l}
u^{\prime}=u \rho-k \\
v^{\prime}=v \rho+A \\
\rho^{\prime}=u^{2}+v^{2}-A .
\end{array}\right.
$$

Then each orbit $t \mapsto(u(t), v(t), \rho(t))$ determines a Ricci soliton metric of the form $g=\left(\mathrm{d} x^{1}\right)^{2}+a(t)^{2}\left(\mathrm{~d} x^{2}\right)^{2}+b(t)^{2}\left(\mathrm{~d} x^{3}\right)^{2}$ via the correspondence: $t=x^{1}, u=b^{\prime} / b$, $v=a^{\prime} / a$. The soliton is shrinking, steady or expanding, when $A<0,=0,>0$, respectively. Up to addition of a Killing vector field, the soliton flow is given by:

$$
E=(\rho+u+v) \frac{\partial}{\partial x^{1}}-(k+A) x^{3} \frac{\partial}{\partial x^{3}}
$$

it is of gradient type if and only if either $k+A=0$, or $b^{\prime}=0(u=0)$.

Proof The components of the Ricci tensor are given by Lemma 2.1(ii). By (10), we have

$$
\begin{aligned}
\mathcal{L}_{E} g & =2 \nabla \mathrm{d} \beta+\mathcal{L}_{\left(\frac{f}{b}\right) \partial_{3}} g \\
& =2 \nabla \mathrm{d} \beta+2 \mathrm{~d}\left(\frac{f}{b}\right)\left(b^{2} \mathrm{~d} x^{3}\right) \\
& =2 \nabla \mathrm{d} \beta+2 b \mathrm{~d} f \mathrm{~d} x^{3}-2 f b^{\prime} \mathrm{d} x^{1} \mathrm{~d} x^{3}
\end{aligned}
$$

where products of 1-forms correspond to symmetric tensor products. Now the second fundamental form $\nabla \mathrm{d} \beta$ decomposes as

$$
\begin{aligned}
\nabla \mathrm{d} \beta= & \left(\partial_{i j}^{2} \beta-\Gamma_{i j}^{k} \partial_{k} \beta\right) \mathrm{d} x^{i} \mathrm{~d} x^{j} \\
= & \partial_{11}^{2} \beta\left(\mathrm{d} x^{1}\right)^{2}+2\left(\partial_{12}^{2} \beta-\frac{a^{\prime}}{a} \partial_{2} \beta\right) \mathrm{d} x^{1} \mathrm{~d} x^{2} \\
& +\left(\partial_{22}^{2} \beta+a a^{\prime} \partial_{1} \beta\right)\left(\mathrm{d} x^{2}\right)^{2}+b b^{\prime} \partial_{1} \beta\left(\mathrm{d} x^{3}\right)^{2}
\end{aligned}
$$


Collecting terms, the equations (5) for a soliton become:

$$
\begin{aligned}
& -\left(u^{\prime}+v^{\prime}+u^{2}+v^{2}\right)\left(\mathrm{d} x^{1}\right)^{2}-a^{2}\left(v_{u}^{\prime} v+v^{2}\right)\left(\mathrm{d} x^{2}\right)^{2}-b^{2}\left(u^{\prime}+u^{2}+u v\right)\left(\mathrm{d} x^{3}\right)^{2} \\
& +\partial_{11}^{2} \beta\left(\mathrm{d} x^{1}\right)^{2}+2\left(\partial_{12}^{2} \beta-\frac{a^{\prime}}{a} \partial_{2} \beta\right) \mathrm{d} x^{1} \mathrm{~d} x^{2}+\left(\partial_{22}^{2} \beta+a a^{\prime} \partial_{1} \beta\right)\left(\mathrm{d} x^{2}\right)^{2} \\
& +b b^{\prime} \partial_{1} \beta\left(\mathrm{d} x^{3}\right)^{2}+\left(b \mathrm{~d} f \mathrm{~d} x^{3}-f b^{\prime} \mathrm{d} x^{1} \mathrm{~d} x^{3}\right)+A\left(\left(\mathrm{~d} x^{1}\right)^{2}+a^{2}\left(\mathrm{~d} x^{2}\right)^{2}\right. \\
& \left.+b^{2}\left(\mathrm{~d} x^{3}\right)^{2}\right)=0
\end{aligned}
$$

with its component parts:

$$
\begin{cases}\text { (i) } & \partial_{12}^{2} \beta-v \partial_{2} \beta=0 \\ \text { (ii) } & -u^{\prime}-v^{\prime}-u^{2}-v^{2}+\partial_{11}^{2} \beta+A=0 \\ \text { (iii) } & -v^{\prime}-u v-v^{2}+\frac{1}{a^{2}} \partial_{22}^{2} \beta+v \partial_{1} \beta+A=0 \\ \text { (iv) } & -\left(u^{\prime}+u^{2}+u v\right) \mathrm{d} x^{3}+u \partial_{1} \beta \mathrm{d} x^{3}+\frac{1}{b} \mathrm{~d} f-\frac{f b^{\prime}}{b^{2}} \mathrm{~d} x^{1}+A \mathrm{~d} x^{3}=0 .\end{cases}
$$

Now the latter equation implies that $\partial f / \partial x^{2}=0$, that

$$
\frac{\partial f}{\partial x^{1}}-f \frac{b^{\prime}}{b}=0
$$

and that

$$
-u^{\prime}-u^{2}-u v+u \partial_{1} \beta+\frac{1}{b} \frac{\partial f}{\partial x^{3}}+A=0 .
$$

On the other hand, these same equations give:

$$
d\left(\frac{f}{b}\right)=\left(u^{\prime}+u^{2}+u v-u \gamma-A\right) \mathrm{d} x^{3}
$$

where we have written $\gamma=\partial_{1} \beta$.

We will now make the assumption that $\gamma$ depends on $x^{1}$ only and that $\partial_{22}{ }^{2} \beta=0$. In fact if we suppose that $u \neq 0$, then since the derivative of the RHS of (16) must vanish, it automatically follows that $\partial_{12}{ }^{2} \beta=0$ and $\gamma$ depends on $x^{1}$ only. If, further, we suppose that $v \neq 0$, then integration of (13)(i) shows that $\partial_{1} \beta-v \beta=\zeta$, for some function $\zeta$ which depends on $x^{1}$ only, so that in fact $\beta=\beta\left(x^{1}\right)$ and once more it is automatic that $\partial_{22}{ }^{2} \beta=0$. However, we do not wish to exclude the possibility that one or other of $u$ and $v$ vanish identically (so that $a$ or $b$ are constant).

Since the derivative of the right-hand side of (16) vanishes, we must have

$$
u^{\prime}+u^{2}+u v-u \gamma-A=\text { const. }
$$


We therefore have the system:

$$
\left\{\begin{array}{r}
-u^{\prime}-v^{\prime}-u^{2}-v^{2}+\gamma^{\prime}+A=0 \\
-v^{\prime}-u v-v^{2}+v \gamma+A=0 \\
u^{\prime}+u^{2}+u v-u \gamma+k=0
\end{array}\right.
$$

for some constant $k$. The substitution $\rho=\gamma-u-v$ now gives the dynamical system (11).

If we now take an orbit $t \mapsto(u(t), v(t), \rho(t))$, then we can construct the soliton flow as follows. We in fact only need to express the partial derivatives $\partial \beta / \partial x^{1}$ and $\partial \beta / \partial x^{2}$. Firstly, $\partial_{1} \beta=\gamma=\rho+u+v$. Now if $v \neq 0$, then (13)(i) shows that $\partial_{2} \beta=0$. On the other hand, if $v \equiv 0$, then the assumptions $\partial_{12}{ }^{2} \beta=0$ and $\partial_{22}{ }^{2} \beta=0$ shows that $\partial_{2} \beta$ is constant. But since in this case we have $a$ constant, $\partial / \partial x^{2}$ is a Killing vector field and we can take $\partial_{2} \beta$ to be zero.

To find $f$, we must solve (14) and (16) (recalling that $\partial f / \partial x^{2}=0$ ). From (16)

$$
\begin{aligned}
\frac{1}{b} \frac{\partial f}{\partial x^{3}} & =u^{\prime}+u^{2}+u v-u \gamma-A \\
& =u^{\prime}-u \rho-A \\
& =-k-A,
\end{aligned}
$$

so that

$$
\frac{\partial f}{\partial x^{3}}=-(k+A) b .
$$

But now from (14), $\frac{\partial}{\partial x^{1}} \ln \left(\frac{f}{b}\right)=0$, so that $f / b=B\left(x^{2}, x^{3}\right)$ for some function $B$ of $x^{2}$ and $x^{3}$ only. Since $\partial f / \partial x^{2}=0, B$ depends only on $x^{3}$ and comparison with (17) shows that

$$
f=-\left((k+A) x^{3}+c\right) b,
$$

where $c$ is some constant. But in fact $f$ is only defined up to addition of a constant multiple of $b$ (see Lemma 3.2 below), so we can take the constant $c$ to be zero.

Finally, the soliton is of gradient type if and only if $E$ is a gradient. But this is the case (on a simply connected manifold) if and only if $\mathrm{d} E^{b}=0$, where $E^{b}=\mathrm{d} \beta+f b \mathrm{~d} x^{3}$. But

$$
\mathrm{d} E^{b}=\left(\frac{\partial f}{\partial x^{1}} b+f b^{\prime}\right) \mathrm{d} x^{1} \wedge \mathrm{d} x^{3},
$$

which vanishes if and only if

$$
\frac{\partial f}{\partial x^{1}} b+f b^{\prime}=2 \frac{\partial f}{\partial x^{1}} b=2 f b^{\prime}=0,
$$


that is, if and only if either $f \equiv 0$, equivalently $k+A=0$, or $b^{\prime}=0$.

Let us make some general remarks, which will be useful in what follows. Firstly, the constant $A$ in (11) can be normalized by permitting a homothetic change of the metric $g$. More precisely, set $s=c t$ ( $c$ constant), so that

$$
g=\frac{\mathrm{d} s^{2}}{c^{2}}+a(t)^{2}\left(\mathrm{~d} x^{2}\right)^{2}+b(t)^{2}\left(\mathrm{~d} x^{3}\right)^{2}=\frac{1}{c^{2}}\left(\mathrm{~d} s^{2}+\widetilde{a}(s)^{2}\left(\mathrm{~d} x^{2}\right)^{2}+\widetilde{b}(s)^{2}\left(\mathrm{~d} x^{3}\right)^{2}\right)
$$

where we have written $\widetilde{a}(s)=c a(s / c), \widetilde{b}(s)=c b(s / c)$. If we define $\widetilde{u}(s)=$ $\widetilde{b}^{\prime}(s) / \widetilde{b}(s)=u(t) / c, \widetilde{v}(s)=\widetilde{a}^{\prime}(s) / \widetilde{a}(s)=v(t) / c$. Then

$$
\widetilde{u}^{\prime}(s)=\frac{1}{c^{2}} u^{\prime}(t)=\frac{1}{c^{2}}(u \rho-k)=\widetilde{u} \widetilde{\rho}-\frac{k}{c^{2}}
$$

where we have defined $\widetilde{\rho}(s)=\rho(s / c) / c$. Similarly for the other derivatives and the dynamical system (11) now becomes:

$$
\left\{\begin{array}{l}
\widetilde{u}^{\prime}(s)=\widetilde{u} \widetilde{\rho}-\frac{k}{c^{2}} \\
\widetilde{v}^{\prime}(s)=\widetilde{v} \widetilde{A}+\frac{A}{c^{2}} \\
\widetilde{\rho}^{2}(s)=\widetilde{u}^{2}+\widetilde{v}^{2}-\frac{A}{c^{2}} .
\end{array}\right.
$$

By choosing $c$ appropriately, we can now normalise the constant $A$ to be either $-2,0,2$. Secondly, the soliton flow restricted to have the form (10), is essentially unique.

Lemma 3.2 Suppose the metric (9) admits a soliton structure derived from the dynamical system (11) with corresponding flow

$$
E=\operatorname{grad} \beta+f U .
$$

Then apart from the trivial Euclidean case when both $a$ and $b$ are constant, $\beta$ is defined up to addition of a constant and $f$ is defined up to the freedom:

$$
f \rightarrow \widetilde{f}=f+c b \quad(c \text { const }) .
$$

When $a$ and $b$ are both constant, we have the additional freedom:

$$
\beta \rightarrow \widetilde{\beta}=\beta+B x^{1}+C \quad(B, C \text { const }) .
$$

Proof That $f$ admits the freedom (19) is obvious from equation (16). On the other hand, suppose the vector fields $E_{1}$ and $E_{2}$ determine the same soliton structure. Then the difference $E=E_{1}-E_{2}$ is a Killing vector field: $\mathcal{L}_{E} g=0$. If we write 
$E_{i}=\operatorname{grad} \beta_{i}+f_{i} U$ and set $\beta=\beta_{1}-\beta_{2}, f=f_{1}-f_{2}$, then $\mathcal{L}_{E} g=0$ is equivalent to the following system:

$$
\begin{cases}\text { (i) } & \partial_{11} \beta=0 \\ \text { (ii) } & \partial_{12} \beta-v \partial_{2} \beta=0 \\ \text { (iii) } & \partial_{22} \beta+a a^{\prime} \partial_{1} \beta=0 \\ \text { (iv) } & u \partial_{1} \beta \mathrm{d} x^{3}+d\left(\frac{f}{b}\right)=0 .\end{cases}
$$

From (ii), we deduce that

$$
\partial_{1} \beta-v \beta=\zeta\left(x^{1}\right)
$$

for some function $\zeta$ which depends on $x^{1}$ only. But now differentiating this with respect to $x^{1}$ and applying the first equation shows that

$$
v^{\prime} \beta+v \partial_{1} \beta+\zeta^{\prime}=0
$$

from which we deduce that

$$
\beta\left(v^{\prime}+v^{2}\right)+v \zeta+\zeta^{\prime}=0
$$

so that, provided $v^{\prime}+v^{2} \neq 0$, the function $\beta$ depends on $x^{1}$ only.

Suppose first that $v^{\prime}+v^{2} \neq 0$. From (20)(iii), we now deduce that either $a^{\prime}=0$ or $\partial_{1} \beta=0$. But if $a^{\prime}=0$ then $v$ vanishes, so we must have $\partial_{1} \beta=0$ and $\beta$ is constant. Then (20)(iv) shows that $f=c b$ for some constant $c$.

Suppose now that $v^{\prime}+v^{2}=0$, but that $a$ is non-constant. Then $v=1 / x^{1}$ up to translation of $x^{1}$. But from (22), $v \zeta+\zeta^{\prime}=0$, which implies that $\zeta=c / x^{1}$ for some constant $c$. Then (21) now implies that $\partial_{1}\left(\beta / x^{1}\right)=c /\left(x^{1}\right)^{2}$, so that

$$
\frac{\beta}{x^{1}}=-\frac{c}{x^{1}}+\mu\left(x^{2}\right)
$$

for some function $\mu$ which depends on $x^{2}$ only, and $\beta=x^{1} \mu-c$. In particular $\partial_{1} \beta=\mu\left(x^{2}\right)$ and (20)(iv) implies that

$$
u \mu \mathrm{d} x^{3}+\mathrm{d}\left(\frac{f}{b}\right)=0 .
$$

On differentiating this, we deduce that both $u^{\prime} \mu=0$ and $u \mu^{\prime}=0$. If $\mu=0$, then $\beta$ is constant and we are done, as above. Otherwise $u^{\prime}=0$. If $u=0$, then the expression $v=1 / x^{1}$ contradicts the dynamical system (11). So $u$ must be a non-zero constant, but then $\mu^{\prime}=0$ and $\beta=\beta\left(x^{1}\right)$ and the previous arguments (with $v^{\prime}+v^{2} \neq 0$ ) apply. 
Suppose finally that $a^{\prime} \equiv 0$, so in particular $v \equiv 0$ and from (21) $\partial_{1} \beta=\zeta\left(x^{1}\right)$. From (20)(i), we must have $\zeta$ constant. We suppose this constant non-zero otherwise $\beta$ is constant as above. Then the exterior derivative of (20)(iv) shows that $u^{\prime} \equiv 0$ and so $u$ is also constant. But we are supposing that the components of the metric satisfy the dynamical system (11). In particular we must have $A=0$ together with $0=\rho u-k$ and $\rho^{\prime}=u^{2}$. But since $u$ is constant this can only occur when $k=0$ and $u=0$. We now have $\partial_{1} \beta=\zeta$ constant, so that $\beta=B x^{1}+C$, for constants $B$ and $C$.

A consequence of the above lemma is that, apart from the trivial Euclidean case when both $a$ and $b$ are constant, distinct orbits of the dynamical system (11) do not arise from the same soliton structure due to the addition of a Killing field to $E$. We may on the other hand have different orbits giving the same soliton structure due to diffeomorphism, for example the diffeomorphism which interchanges $x^{2}$ and $x^{3}$ gives an example.

Before going on to study the dynamical system (11), we give without proof, the dynamical system that occurs with the more general form of the metric (8). This can be deduced by applying the ansatz given by [1, Theorem 2], or by direct calculation, which is rather involved.

Theorem 3.3 Let $A$ and $k$ be constants and consider the 5-dimensional autonomous dynamical system:

$$
\left\{\begin{array}{l}
u^{\prime}=u \rho+\delta-k \\
v^{\prime}=v \rho-\delta+A \\
\rho^{\prime}=u^{2}+v^{2}+\delta-A \\
\delta^{\prime}=2 \delta \zeta \\
\zeta^{\prime}=2\left(u^{2}+v^{2}-u v\right)+2 \rho(v-u)-\zeta^{2}+\rho \zeta-\delta+A+2 k
\end{array}\right.
$$

Then each orbit $t \mapsto(u(t), v(t), \rho(t), \delta(t), \zeta(t))$ in the half space $\delta \geq 0$ determines a Ricci soliton metric of the form (8) via the correspondence: $t=x^{1}, u=b^{\prime} / b, v=a^{\prime} / a$, $\delta=\frac{1}{2}\left(\frac{b}{a}\left(\frac{e}{b}\right)^{\prime}\right)^{2}$. The soliton is shrinking, steady or expanding, when $A<0,=0,>0$, respectively.

Note that, for an orbit to correspond to a soliton we must have $\delta \geq 0$; in which case $\delta \equiv 0$ if and only if the horizontal distribution is integrable and we are back in the case of system (11). Furthermore, if $\delta$ vanishes at any point along an orbit, then it is identically zero along that orbit. This is a consequence of the uniqueness of the solution of the ODE: $\delta^{\prime}=2 \delta \zeta$ for a given initial condition. Thus each orbit lies in the half-space $\delta>0$, the half-space $\delta<0$, or the hyper-plane $\delta=0$. 
A further observation is that there are no fixed points in the half-space $\delta>0$. For such a fixed point would require $\zeta=0$ and

$$
\left\{\begin{array}{l}
0=2\left(u^{2}+v^{2}-u v\right)+2 \rho(v-u)-\delta+A+2 k \\
0=u^{2}+v^{2}+\delta-A \\
0=u \rho+\delta-k \\
0=v \rho-\delta+A
\end{array}\right.
$$

Substitution of the last three of these equation into the first yields

$$
0=-2 u v+\delta+A \text {. }
$$

Then the second gives

$$
0=(u-v)^{2}+2 \delta
$$

which cannot be satisfied for $\delta>0$.

Remark 3.4 We emphasize that (11) and (23) do not give all solitons with metrics of the form (8), but only those with $X=\operatorname{grad} \beta$ restricted as in the proof of Theorem 3.1. For example, the geometry Nil has soliton flow determined by the function $\beta\left(x^{1}, x^{2}\right)=-\frac{1}{2}\left(\left(x^{1}\right)^{2}+\left(x^{2}\right)^{2}\right)$ whose dependence on the variable $x^{2}$ means that it doesn't occur in our class of examples.

\section{The phase space of the dynamical system}

Our aim in this section is to make some preliminary observations about the dynamical system (11) and discuss some well know soliton metrics that arise. In the next section we will undertake a more thorough phase space analysis and deduce some new examples.

The first remark is that the dynamical system (11) can be viewed as a gradient system, for if we set $\sigma=\rho / \sqrt{2}$, then it becomes:

$$
\left\{\begin{aligned}
u^{\prime} & =\sqrt{2} \sigma u-k \\
v^{\prime} & =\sqrt{2} \sigma v+A \\
\sigma^{\prime} & =\frac{1}{\sqrt{2}}\left(u^{2}+v^{2}-A\right)
\end{aligned}\right\}=\operatorname{grad} F
$$

where $F(u, v, \sigma)=\frac{\sigma}{\sqrt{2}}\left(u^{2}+v^{2}-A\right)-k u+A v$. Provided $A \neq 0$, equilibrium points are given by

$$
\left( \pm \frac{k \sqrt{A}}{\sqrt{k^{2}+A^{2}}}, \mp \frac{A \sqrt{A}}{\sqrt{k^{2}+A^{2}}}, \pm \sqrt{\frac{k^{2}+A^{2}}{2 A}}\right)
$$


in particular, if $A<0$ (the soliton is shrinking), there are none. If $A>0$, then both equilibrium points are unstable. If $A=0$, then for an equilibrium point, we must have $k=0$ in which case the entire axis $u=v=0$ consists of equilibrium points.

For a given $c$, the intersection of the level $F=c$ with the plane $\sigma=\sigma_{0}$, is a circle centre:

$$
\left(\frac{k}{\sqrt{2} \sigma_{0}},-\frac{A}{\sqrt{2} \sigma_{0}}, \sigma_{0}\right)
$$

of radius: $\sqrt{\frac{\sqrt{2} c}{\sigma_{0}}+\frac{\left(k^{2}+A^{2}\right)}{2 \sigma_{0}^{2}}+A}$. At the equilibrium points, the value of $F$ is given by $\mp \sqrt{A} \sqrt{k^{2}+A^{2}}$ and the radius degenerates to zero. At points along the curve (26) given by the centres of the circles, we have $\operatorname{grad} F$ is vertical (parallel to the $\sigma$-axis).

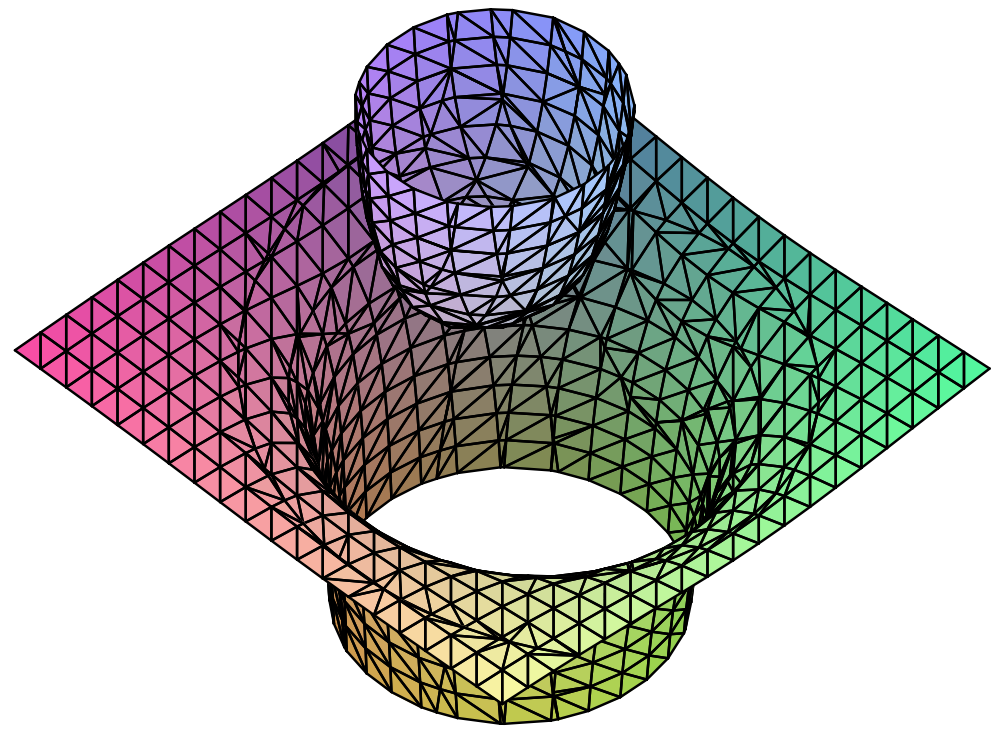

Figure 1: Graph of the level $F=-10$ with $k$ and $A$ both positive. 
A useful subclass of orbits is given by the following lemma.

Lemma 4.1 Any orbit passing through the axis $u=v=0$ lies in the plane: $A u+k v=$ 0 . In particular, this plane is an invariant subspace.

Proof Consider the evolution of the quantity $A u+k v$ along a trajectory:

$$
\frac{\mathrm{d}}{\mathrm{d} t}(A u+k v)=A u^{\prime}+k v^{\prime}=\sqrt{2} \sigma(A u+k v) .
$$

Thus if $A u+k v=0$ at $t=0$, then $A u+k v$ remains zero by the uniqueness of the solution of an ODE for a given initial condition. But along the axis $u=v=0$, the quantity $A u+k v=0$.

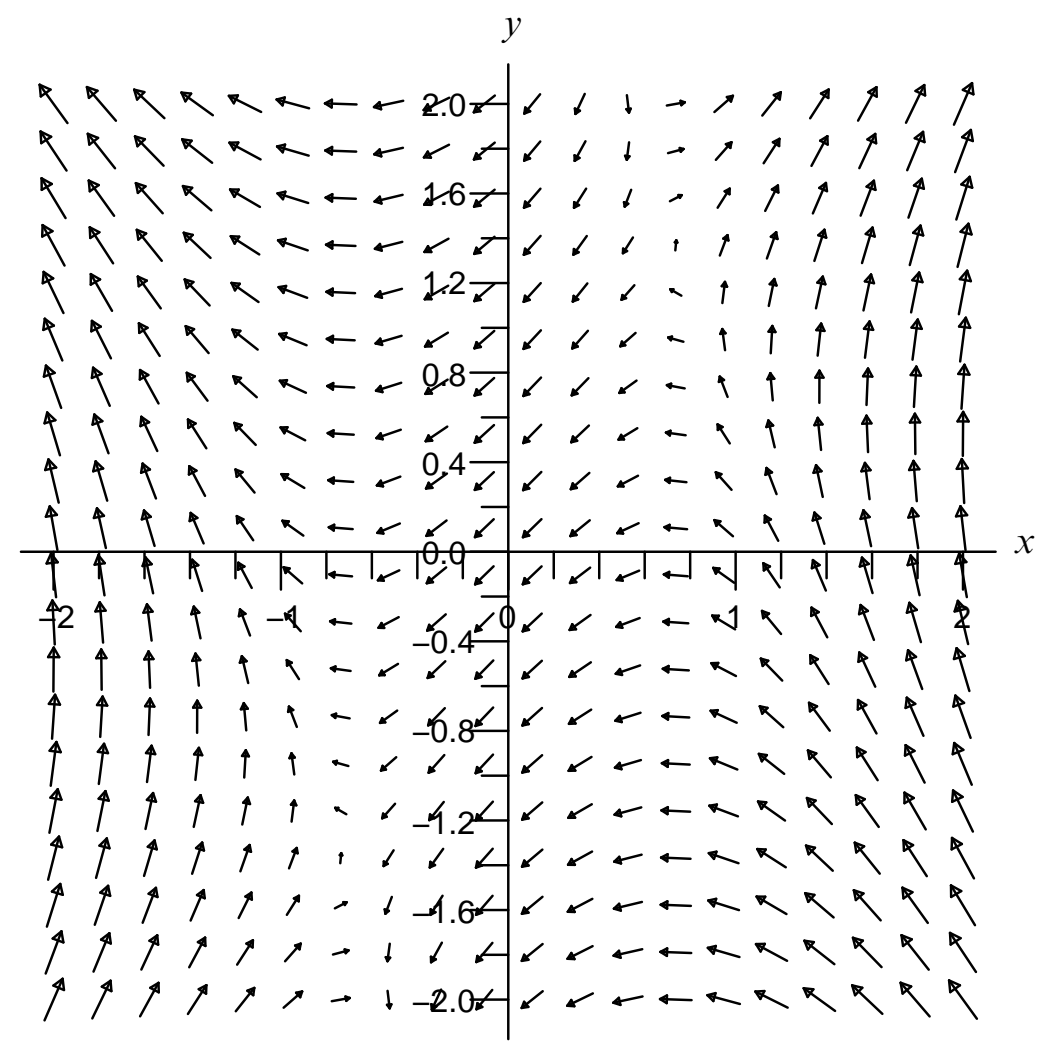

Figure 2: Phase portrait restricted to the invariant plane $A u+k v=0$.

Our new examples will be given by the orbit passing through the origin. In the first instance it is important to know that in general this doesn't encounter an equilibrium point. 
Proposition 4.2 Let $A>0$ and $|k|>0$. Then the orbit passing through the origin never encounters an equilibrium point.

Proof Once more, it is convenient to work with the system (24). The tangent to the orbits is in the direction of $\operatorname{grad} F$. We therefore compare the direction of $\operatorname{grad} F$ with the direction of rays from the origin at each point. More precisely, we will compare the gradients of these two vectors with respect to the $\sigma=$ const. planes at each point. For the ray, at the point $(u, v, \sigma)$, it is given by the number

$$
G(\text { ray }, \sigma)=\frac{\sigma}{\sqrt{u^{2}+v^{2}}}
$$

and for $\operatorname{grad} F$, it is given by the number

$$
G(\operatorname{grad} F, \sigma)=\frac{\left(u^{2}+v^{2}-A\right) / \sqrt{2}}{\left\{2 \sigma^{2}\left(u^{2}+v^{2}\right)+2 \sqrt{2} \sigma(-k u+A v)+k^{2}+A^{2}\right\}^{1 / 2}} .
$$

Then $|G(\operatorname{grad} F, \sigma)| \leq \mid G($ ray, $\sigma) \mid$ if and only if

$$
\left(u^{2}+v^{2}-A\right)^{2}\left(u^{2}+v^{2}\right) \leq 2 \sigma^{2}\left\{2 \sigma^{2}\left(u^{2}+v^{2}\right)-2 \sqrt{2} \sigma(-k u+A v)+k^{2}+A^{2}\right\} .
$$

We can parameterise the ray joining the origin with the equilibrium point $\left(-\frac{k \sqrt{A}}{\sqrt{k^{2}+A^{2}}},+\frac{A \sqrt{A}}{\sqrt{k^{2}+A^{2}}},-\sqrt{\frac{k^{2}+A^{2}}{2 A}}\right)$ in the form:

$$
s \mapsto\left(-s \frac{k \sqrt{A}}{\sqrt{k^{2}+A^{2}}},+s \frac{A \sqrt{A}}{\sqrt{k^{2}+A^{2}}},-s \sqrt{\frac{k^{2}+A^{2}}{2 A}}\right) .
$$

Then along this ray, the inequality $(27)$ reads:

$$
s^{2}\left(s^{2}-1\right)^{2} A^{4} \leq s^{2}\left(s^{2}-1\right)\left(k^{2}+A^{2}\right)^{2}
$$

which is always satisfied. Furthermore, the inequality is strict except when $s=0,1$. The conclusion is that along the ray joining the origin to the equilibrium point, the vector field $\operatorname{grad} F$ has a less steep incline, so the orbit falls above the ray and remains there. For negative time, by symmetry, the orbit lies below the other equilibrium point and remains there. We recall that by Lemma 4.1, the orbit remains in the plane $A u+k v=0$, which also contains the equilibrium points.

Also, note that if $k$ is positive and $u$ is initially negative along a trajectory, then it remains so, for if $u=0$, then $u^{\prime}=-k<0$. Similarly, if $v>0$ initially, then it remains so, ie in future time the trajectory remains in the quadrant $u<0, v>0$. Similary, in past time, the trajectory is in the quadrant $u>0, v<0$. Similar reasoning restricts the solution to particular quadrants when $k<0$. In conclusion, the orbit through the 
origin avoids the equilibrium points. We remark that the above analysis of the orbit can quickly be verified by performing a Maple plot of the solution curve, see Figure 2.

We now wish to explore further properties of the soliton corresponding to the orbit through the origin. We can consider it as a solution to the complex system:

$$
\left\{\begin{array}{l}
w^{\prime}=w \rho-k+\mathrm{i} A \\
\rho^{\prime}=|w|^{2}-A
\end{array}\right.
$$

where we set $w(t)=u(t)+\mathrm{i} v(t)$ and for the moment we do not restrict the constant $A$. From the theory of first order differential equations of the type

$$
X^{\prime}(t)=F(t, X(t))
$$

if $F$ is analytic, then so is each solution (see, for example Dieudonné [5, (10.5.3)]). In order to show that the soliton is neither homogeneous, nor one of the other known types in the list given in the Introduction, we will expand the solution as a power series in $t$ about the origin and calculate its curvature. We consider the more general case of an orbit passing through the point $(w, \rho)=(0, c)$. So write:

$$
w=\sum_{n=0}^{\infty} \alpha_{n} t^{n}, \quad \rho=\sum_{n=0}^{\infty} c_{n} t^{n} .
$$

On substituting into (29), we obtain the following recurrence relations for the coefficients:

$$
\begin{aligned}
& \alpha_{n+1}=\frac{1}{n+1} \sum_{k+l=n} \alpha_{k} c_{l} \quad(n \geq 1) \alpha_{0}=0, \alpha_{1}=-k+\mathrm{i} A \\
& c_{n+1}=\frac{1}{n+1} \sum_{k+l=n} \alpha_{k} \bar{\alpha}_{l} \quad(n \geq 1) c_{0}=c, c_{1}=-2 .
\end{aligned}
$$

It is now possible to calculate successive coefficients to obtain:

$$
\begin{gathered}
w=(-k+\mathrm{i} A)\left(t+\frac{c}{2} t^{2}+\frac{1}{3 \cdot 2}\left(-2 A+c^{2}\right) t^{3}-\frac{c}{6 \cdot 4}\left(5 A+c^{2}\right) t^{4}+\right. \\
\left.\frac{1}{6 \cdot 5 \cdot 4}\left(8 k+16 A^{2}-9 A c-c^{4}\right) t^{5}+\mathcal{O}\left(t^{6}\right)\right) \\
\rho=c-A t+\left(k^{2}+A^{2}\right)\left(\frac{1}{3} t^{3}+\frac{c}{4} t^{4}+\frac{1}{5 \cdot 4 \cdot 3}\left(-8 A+7 c^{2}\right) t^{5}+\mathcal{O}\left(t^{6}\right)\right) .
\end{gathered}
$$

The Ricci curvature is now given by Lemma 2.1:

$$
\begin{aligned}
R_{11} & =(k-A)+c(k-A) t+\left(\frac{1}{2}(k-A)\left(-2 A+c^{2}\right)-k^{2}-A^{2}\right) t^{2}+\mathcal{O}\left(t^{3}\right) \\
\frac{1}{a^{2}} R_{22} & =-A-A c t-\left(\frac{1}{2} A\left(-2 A+c^{2}\right)-A(k-A)\right) t^{2}+\mathcal{O}\left(t^{3}\right) \\
\frac{1}{b^{2}} R_{33} & =k+c k t+\left(\frac{1}{2} k\left(-2 A+c^{2}\right)-k(k-A)\right) t^{2}+\mathcal{O}\left(t^{3}\right) .
\end{aligned}
$$


We note that $\frac{k}{a^{2}} R_{22}+\frac{A}{b^{2}} R_{33}=0$. Finally, the scalar curvature is given by the trace of the Ricci curvature:

$$
\mathrm{Scal}=2(k-A)+2 c(k-A) t+\left(c^{2}(k-A)-2 k^{2}\right) t^{2}+\mathcal{O}\left(t^{3}\right)
$$

For the orbit passing through the origin, we put $c=0$ in the above formulae, in which case, both $w$ and $\rho$ become odd functions, whereas the components of the Ricci tensor and the scalar curvature become even functions. We can now establish the non-triviality of the soliton which corresponds to the orbit passing through the origin.

Theorem 4.3 Provided the constants $k$ and $A$ are both non-vanishing with $k+A \neq 0$, the soliton determined by the orbit passing though the origin of the system (11) is neither homogeneous, rotationally symmetric, nor of product type $\Sigma \times \mathbf{R}$, where $\Sigma$ is a 2-dimensional soliton.

Proof Any homogeneous example must have constant scalar curvature. But the expression for the scalar curvature given by (32) shows that this can only happen when $k=0$. To be rotationally symmetric, two of the eigenvalues of the Ricci tensor must coincide. But an inspection of (31) shows that this can only happen when $k+A=0$. Finally, to be of product type, one of the eigenvalues of the Ricci tensor must vanish. It appears from (31) that $R_{11}$ vanishes when $k=A$. However, this is because due to the cumbersome nature of the expression, we have only developed it up to order 2 in $t$. In fact, when $c=0$, the coefficient of $t^{4}$ is equal to

$$
\frac{1}{6 \cdot 4}(k-A)\left(8 k+16 A^{2}\right)+\frac{2}{3} A\left(k^{2}+A^{2}\right)
$$

which shows that $R_{11}$ can only vanish when $k=A=0$.

The above theorem shows that the soliton corresponding to the orbit passing through the origin does not fall into the list of examples given in the Introduction. It is similarly the case when we take non-zero values of $c$. In view of the normalisation of the constant $A$ discussed above, when $A$ is non-constant it is reasonable to set $A=\lambda c^{2}$ in the expansions (30) to give us a family of solitons depending on the two parameters $k$ and $\lambda$. However, it is difficult to know whether some members of this family could be equivalent by diffeomorphism.

Having established the non-triviality of one of the solitons given by the dynamical system (11), let us now look how some known solitons arise. 
Theorem 4.4 When $A>0$, the equilibrium points of the dynamical system (11), correspond to a family of soliton metrics of the form:

$$
\left(\begin{array}{ccc}
1 & 0 & 0 \\
0 & e^{2 t} & 0 \\
0 & 0 & e^{-2 \lambda t}
\end{array}\right)
$$

where $\lambda$ is an arbitrary parameter. In the case when $\lambda=1$ this gives the metric for the geometry Sol, in particular, Sol is one of a 1-parameter family of soliton metrics.

Proof The equilibrium points are given by (25). This gives the metric

$$
\left(\begin{array}{ccc}
1 & 0 & 0 \\
0 & e^{2 A \sqrt{\frac{A}{k^{2}+A^{2}}} t} & 0 \\
0 & 0 & e^{-2 k \sqrt{\frac{A}{k^{2}+A^{2}}} t}
\end{array}\right) .
$$

But under normalisation of the constant $A$, we may suppose $A \sqrt{\frac{A}{k^{2}+A^{2}}}=1$. Now write $\lambda=k / A$ to give the form of the statement of the theorem.

Example 4.5 (Constant curvature examples) The sphere $S^{3}$ arises by setting $A=-2$, $k=2, a=\sin t$ and $b=\cos t$. Then $u=-\tan t, v=\cot t$ and the corresponding orbit of (11) is given by setting $\rho=\frac{1}{\sin t \cos t}-2 \cot t$.

In a similar fashion, hyperbolic 3 -space occurs by setting $A=2, k=-2, a=\sinh t$ and $b=\cosh t$. Then $u=\tanh t, v=\operatorname{coth} t$ and the corresponding orbit occurs by setting $\rho=-\frac{1}{\sinh t \cosh t}-2 \tanh t$.

Note that in both cases, the orbits are not defined for all time. Also, in both cases, with reference to Theorem 4.3, we have $k+A=0$.

Example 4.6 (Product examples) Examples of the form $\Sigma \times \mathbf{R}$ occur when $b \equiv 1$. Then $u \equiv 0$ and we obtain the 2-dimensional dynamical system:

$$
\left\{\begin{array}{l}
v^{\prime}=v \rho+A \\
\rho^{\prime}=v^{2}-A .
\end{array}\right.
$$

But this is precisely the system that occurs for the construction of a 2-dimensional Ricci soliton with metric of the form: $h=\left(\mathrm{d} x^{1}\right)^{2}+a\left(x^{1}\right)^{2}\left(\mathrm{~d} x^{2}\right)^{2}$. For example, Hamilton's 2-dimensional cigar is given by the metric: $h=\left(\mathrm{d} x^{1}\right)^{2}+\tanh ^{2} t\left(\mathrm{~d} x^{2}\right)^{2}$. Then it is easily seen that the above equations are verified with $A=0$. This example is a complete steady gradient soliton; for more details, see for example Chow-Knopf [4]. 
Example 4.7 (Warped product examples) We consider the special case when $a \equiv b$, which can be considered as a warped product of the real line with standard Euclidean 2 -space. Now our system can be viewed as the sub-system of the dynamical system (11) given by the orbits passing through the axis $u=v=0$ when $A+k=0$ (recall that by Lemma 4.1 , these all lie in the plane $u=v$ ). This then leads to the 2-dimensional dynamical system:

$$
\left\{\begin{array}{l}
u^{\prime}=u \rho+A \\
\rho^{\prime}=2 u^{2}-A .
\end{array}\right.
$$

The behaviour of solutions is exactly as for the system (29) discussed above. By eliminating $\rho$, we see that $u$ is given as a solution to the second order ODE:

$$
u^{\prime \prime} u-\left(u^{\prime}\right)^{2}+A u^{\prime}+A u^{2}-2 u^{4}=0 .
$$

A particular solution when $A=0$ is given by $u= \pm \frac{1}{\sqrt{2}} t^{-1}$, which yields the metric $g=d t^{2}+t^{ \pm \sqrt{2}}\left(\left(\mathrm{~d} x^{2}\right)^{2}+\left(\mathrm{d} x^{3}\right)^{2}\right)$. This singular metric of negative scalar curvature was also noticed in [1]. It corresponds to a steady gradient 3-dimensional soliton. If we don't insist on completeness, this example provides an answer to [3, Problem 1.88]; by Lemma 2.1, we see that the Ricci tensor is positive whatever sign is taken in the expression for $u$, as is required - cf the discussion in [3].

\section{Asymptotic analysis}

In this section we concentrate on the orbit of the dynamical system (11) that passes through the origin and study its asymptotic behaviour. This will enable us to understand better the soliton metric that it produces.

Firstly, let us make the orthogonal substitution:

$$
\xi=\frac{A u+k v}{\sqrt{k^{2}+A^{2}}} \quad \eta=\frac{-k u+A v}{\sqrt{k^{2}+A^{2}}}
$$

which puts (11) into the form:

$$
\left\{\begin{array}{l}
\xi^{\prime}=\xi \rho \\
\eta^{\prime}=\eta \rho+\sqrt{k^{2}+A^{2}} \\
\rho^{\prime}=\xi^{2}+\eta^{2}-A .
\end{array}\right.
$$

Note that the sign in front of the square root $\sqrt{k^{2}+A^{2}}$ can be compensated for by replacing $\eta$ with $-\eta$. We study the orbit with initial condition $(\xi(0), \eta(0), \rho(0))=$ 
$(0,0,0)$, so that $\xi(t)=0$ for all $t$, and we now have the reduced system:

$$
\left\{\begin{array}{l}
\eta^{\prime}=\eta \rho+\sqrt{k^{2}+A^{2}} \\
\rho^{\prime}=\eta^{2}-A
\end{array}\right.
$$

If $A>0$, the fixed points are given by $\left( \pm \sqrt{A}, \mp \sqrt{\frac{k^{2}+A^{2}}{A}}\right)$, otherwise there are none unless $A=k=0$ in which case the whole axis $\eta=0$ consists of fixed points. We study the different cases.

Case (i) $A>0, k=0$ We now have the system

$$
\left\{\begin{array}{l}
\eta^{\prime}=\eta \rho+A \\
\rho^{\prime}=\eta^{2}-A
\end{array}\right.
$$

By uniqueness, the solution through $(0,0)$ satisfies $\rho=-\eta$, and we have the single equation $\eta^{\prime}=-\eta^{2}+A$ with condition $\eta(0)=0$. The solution is given by $\eta(t)=\sqrt{A} \tanh \sqrt{A} t$. So it exists for all time and joins the fixed points. To find the corresponding metric, we note that $u \equiv 0$, so that $b(t)$ is constant; then we solve $\eta=v=a^{\prime} / a$ to give $a(t)=\sinh \sqrt{A} t$. This gives the metric

$$
\left(g_{i j}\right)=\left(\begin{array}{ccc}
1 & 0 & 0 \\
0 & \sinh ^{2} \sqrt{A} t & 0 \\
0 & 0 & b
\end{array}\right)
$$

which corresponds to the product $H^{2} \times \mathbf{R}$.

Case (ii) $A>0, k \neq 0 \quad$ The first observation is that the orbit starting at $(0,0)$ enters the slice $\eta+\rho>0, \eta-\rho>0$ and remains there. For

$$
(\eta+\rho)^{\prime}=\eta(\eta+\rho)+\sqrt{k^{2}+A^{2}}-A ;
$$

so that since $(\eta+\rho)^{\prime}(0)>0$, initially $\eta+\rho$ becomes positive. Suppose at some future time $t>0$ we have $(\eta+\rho)(t)=0$ and let $t_{1}>0$ be the first such time; then $(\eta+\rho)^{\prime}\left(t_{1}\right)=\sqrt{k^{2}+A^{2}}-A>0$ : a contradiction, since we must have $(\eta+\rho)^{\prime}\left(t_{1}\right) \leq 0$. Thus $(\eta+\rho)(t)>0$ for all $t>0$. Similarly. we have

$$
(\eta-\rho)^{\prime}=-\eta(\eta-\rho)+\sqrt{k^{2}+A^{2}}+A
$$

and the same arguments apply. However, we wish to be much more precise in localising the orbit.

Lemma 5.1 The orbit of (34) passing through $(0,0)$ enters the region $\rho>0, \eta>\sqrt{A}$, after which both $\eta$ and $\rho$ increase monotonically and approach infinity. 
Proof To see this, we compare the orbit with the solution given above for the case when $k=0$, so let

$$
\left\{\begin{array}{l}
\widetilde{\eta}=\eta-\sqrt{A} \tanh \sqrt{A} t \\
\widetilde{\rho}=\rho+\sqrt{A} \tanh \sqrt{A} t .
\end{array}\right.
$$

Then

$$
\left\{\begin{array}{l}
\widetilde{\eta}=\widetilde{\eta \rho}+\sqrt{A} \tanh \sqrt{A} t(\widetilde{\rho}-\widetilde{\eta})+\sqrt{k^{2}+A^{2}}-A \\
\widetilde{\rho}=\widetilde{\eta}^{2}+2 \sqrt{A} \widetilde{\eta} \tanh \sqrt{A} t .
\end{array}\right.
$$

Now $\widetilde{\eta}(0)=\widetilde{\rho}(0)=0, \widetilde{\eta}^{\prime}(0)>0, \widetilde{\rho}^{\prime}(0)=\widetilde{\rho}^{\prime \prime}(0)=0$ and $\widetilde{\rho}^{\prime \prime \prime}(0)=2 \widetilde{\eta}^{\prime}(0)^{2}+$ $\frac{4 A \widetilde{\eta}^{\prime}(0)}{\cosh ^{2} \sqrt{A} t}>0$. So that both $\widetilde{\eta}$ and $\widetilde{\rho}$ increase initially.

Now suppose that $\widetilde{\eta}(t)$ vanishes for some $t>0$ and let $t_{0}>0$ be the first time this occurs. Since $\widetilde{\eta}(t) \geq 0$ for $0 \leq t \leq t_{0}, \widetilde{\rho}^{\prime}(t) \geq 0$ for $0 \leq t \leq t_{0}$ which implies that $\widetilde{\rho}(t) \geq 0$ for $0 \leq t \leq t_{0}$ and furthermore, $\widetilde{\rho}\left(t_{0}\right)>0$ (since $\widetilde{\rho}^{\prime \prime \prime}(0)>0$ ). But now

$$
\widetilde{\eta}^{\prime}\left(t_{0}\right)=\sqrt{A} \tanh \sqrt{A} t_{0} \widetilde{\rho}\left(t_{0}\right)+\sqrt{k^{2}+A^{2}}-A>0 ;
$$

a contradiction. Thus $\widetilde{\eta}(t)>0$ for $t>0$ and $\eta(t) \geq \sqrt{A} \tanh \sqrt{A} t$ for all $t \geq 0$. In particular, there exists $t_{1}$ such that for $t \geq t_{1}$, we have $\eta(t) \geq \sqrt{A} / 2$. But then

$$
\widetilde{\rho}^{\prime}(t)=\widetilde{\eta}^{2}(t)+2 \sqrt{A} \widetilde{\eta} \tanh \sqrt{A} t \geq \frac{A}{4}+\frac{A}{2}
$$

for all $t \geq t_{1}$. Thus there exists $t_{2}>0$ such that $\rho(t)>0$ for all $t>t_{2}$. In particular

$$
\begin{aligned}
\eta^{\prime}(t) & =\eta(t) \rho(t)+\sqrt{k^{2}+A^{2}} \\
& \geq \rho(t) \sqrt{A} \tanh \sqrt{A} t+\sqrt{k^{2}+A^{2}} \\
& \geq \sqrt{k^{2}+A^{2}}
\end{aligned}
$$

for all $t>t_{2}$. So necessarily, $\eta(t)$ eventually increases beyond $\sqrt{A}$. But when $\eta>\sqrt{A}$ and $\rho>0$, both $\eta^{\prime}$ and $\rho^{\prime}$ are positive and $\eta$ and $\rho$ both increase monotonically and indefinitely.

We now study the asymptotic behaviour of the orbit $(\eta(t), \rho(t))$ passing though the origin.

Lemma 5.2 There exists a time $T>0$ such that $\lim _{t \nearrow T} \rho(t)=+\infty$ and $\lim _{t \nearrow T} \eta(t)=$ $+\infty$. Furthermore, for $t$ sufficiently large,

$$
\frac{A^{3 / 2}}{\sqrt{k^{2}+A^{2}}} \operatorname{coth} \sqrt{\frac{k^{2}+A^{2}}{A}}(T-t)<\rho(t)<\sqrt{A} \operatorname{coth}(\sqrt{A}(T-t))
$$


(36)

$$
\left(k^{2}+A^{2}\right)^{1 / 4} \cot \left(\left(k^{2}+A^{2}\right)^{1 / 4}(T-t)\right)<\eta(t)<\sqrt{\frac{k^{2}+A^{2}}{A}} \cot (\sqrt{A}(T-t)) .
$$

Proof We suppose that $t_{1}$ is sufficiently large that $\rho\left(t_{1}\right)>\sqrt{A}$, so that both $\rho(t)$ and $\eta(t)$ increase monotonically for $t>t_{1}$. Since $\eta(t)>\rho(t)$, we have $\mathrm{d} \rho / \mathrm{d} t>\rho^{2}-A$. Let $\rho_{0}(t)$ satisfy the differential equation $\frac{\mathrm{d} \rho_{0}}{\mathrm{~d} t}=\rho_{0}(t)^{2}-A$ with initial condition $\rho_{0}\left(t_{1}\right)=\rho\left(t_{1}\right)$. Then this is solved by

$$
\rho_{0}(t)=\frac{\sqrt{A}\left(1+B e^{2 \sqrt{A} t}\right)}{1-B e^{2 \sqrt{A} t}},
$$

with $B$ chosen to satisfy the initial condition, so that

$$
B=\frac{\rho\left(t_{1}\right)-\sqrt{A}}{\rho\left(t_{1}\right)+\sqrt{A}} e^{-2 \sqrt{A} t_{1}}>0 .
$$

But now $\rho_{0}(t) \rightarrow \infty$ in finite time and

$$
\frac{\mathrm{d}}{\mathrm{d} t}\left(\rho-\rho_{0}\right)>\left(\rho^{2}-\rho_{0}^{2}\right)=\left(\rho-\rho_{0}\right)\left(\rho+\rho_{0}\right)
$$

shows that $\rho-\rho_{0}$ initially increases after $t_{1}$, after which it increases monotonically, so there exists a $T<\infty$ with $\lim _{t \nearrow T} \rho(t)=\infty$.

With this established, we can get a more precise estimate on the asymptotic behaviour of $\rho(t)$.

The inequality $\frac{\mathrm{d} \rho}{\mathrm{d} t}>\rho^{2}-A$ is equivalent to $\frac{\mathrm{d}}{\mathrm{d} t} \ln \left(\frac{\rho-\sqrt{A}}{\rho+\sqrt{A}}\right)>2 \sqrt{A}$. Suppose that $t_{1}<t<T$. Integration from $t$ to $T$ now gives

$$
\lim _{s \nearrow T} \ln \left(\frac{\rho(s)-\sqrt{A}}{\rho(s)+\sqrt{A}}\right)-\ln \left(\frac{\rho(t)-\sqrt{A}}{\rho(t)+\sqrt{A}}\right)>2 \sqrt{A}(T-t)
$$

that is

$$
\ln \left(\frac{\rho(t)+\sqrt{A}}{\rho(t)-\sqrt{A}}\right)>2 \sqrt{A}(T-t),
$$

which implies that $\rho(t)<\sqrt{A} \operatorname{coth}(\sqrt{A}(T-t))$.

To obtain a lower bound on $\rho(t)$ as $t \rightarrow+\infty$, we claim that

$$
\sqrt{k^{2}+A^{2}} \rho(t)>A \eta(t),
$$


for $t$ sufficiently large. To see this, let us calculate the derivative

$$
\left(\sqrt{k^{2}+A^{2}} \rho-A \eta\right)^{\prime}=\eta\left(\sqrt{k^{2}+A^{2}} \eta-A \rho\right)-2 A \sqrt{k^{2}+A^{2}} .
$$

Now since $k \neq 0, \sqrt{k^{2}+A^{2}}=A+\varepsilon$ for some $\varepsilon>0$, so that

$$
\eta\left(\sqrt{k^{2}+A^{2}} \eta-A \rho\right)=\varepsilon \eta^{2}+A \eta(\eta-\rho)
$$

which, since $\eta(t)>\rho(t)$ clearly tends to infinity, but this means also that $\sqrt{k^{2}+A^{2}} \rho-$ $A \eta$ becomes arbitrarily large and positive, so that, for $t$ sufficiently large we have $\sqrt{k^{2}+A^{2}} \rho-A \eta>0$, as required. Furthermore the inequality

$$
\rho<\eta<\frac{\sqrt{k^{2}+A^{2}}}{A} \rho
$$

shows that $\lim _{t \nearrow T} \eta(t)=+\infty$ also. We now apply this inequality in a similar way to the above. Thus

$$
\rho^{\prime}=\eta^{2}-A<\left(\frac{k^{2}+A^{2}}{A^{2}}\right) \rho^{2}-A
$$

for $t$ sufficiently large; equivalently

$$
\frac{\mathrm{d}}{\mathrm{d} t} \ln \left(\frac{\sqrt{k^{2}+A^{2}} \rho-A^{3 / 2}}{\sqrt{k^{2}+A^{2}} \rho+A^{3 / 2}}\right)<2 \sqrt{\frac{k^{2}+A^{2}}{A}} .
$$

Integration from $t$ to $T$ now gives the required lower bound on $\rho(t)$.

To establish the asymptotic behaviour of $\eta$, we employ similar tactics. Thus

$$
\eta^{\prime}=\eta \rho+\sqrt{k^{2}+A^{2}}<\eta^{2}+\sqrt{k^{2}+A^{2}}
$$

for $t>t_{1}$, so that

$$
\frac{\mathrm{d}}{\mathrm{d} t} \arctan \left(\frac{\eta}{\left(k^{2}+A^{2}\right)^{1 / 4}}\right)<\left(k^{2}+A^{2}\right)^{1 / 4} .
$$

Integration from $t$ to $T$ now gives

$$
\arctan \left(\frac{\eta(t)}{\left(k^{2}+A^{2}\right)^{1 / 4}}\right)>\frac{\pi}{2}-\left(k^{2}+A^{2}\right)^{1 / 4}(T-t)
$$

and $\eta(t)>\left(k^{2}+A^{2}\right)^{1 / 4} \cot \left(\left(k^{2}+A^{2}\right)^{1 / 4}(T-t)\right)$.

On the other hand, inequality (37) gives

$$
\eta^{\prime}=\eta \rho+\sqrt{k^{2}+A^{2}}>\frac{A \eta^{2}}{\sqrt{k^{2}+A^{2}}}+\sqrt{k^{2}+A^{2}},
$$


and we have

$$
\frac{\mathrm{d}}{\mathrm{d} t} \arctan \left(\frac{\sqrt{A} \eta}{\sqrt{k^{2}+A^{2}}}\right)>\sqrt{A} .
$$

We now proceed similarly to obtain an upper bound on $\eta(t)$.

Corollary 5.3 As $t \nearrow T$, we have

$$
\rho(t)=\frac{c_{1}}{T-t}+\mathcal{O}(T-t), \quad \eta(t)=\frac{c_{2}}{T-t}+\mathcal{O}(T-t),
$$

where $c_{1}$ and $c_{2}$ are constants satisfying

$$
\frac{A^{2}}{k^{2}+A^{2}}<c_{1} \leq 1, \quad 1 \leq c_{2}<\frac{\sqrt{k^{2}+A^{2}}}{A} \text {. }
$$

Proof This follows immediately from the expansions for $\cot x$ and $\operatorname{coth} x$, namely:

$$
\begin{aligned}
\cot x & =\frac{1}{x}\left(1-\frac{x^{2}}{3}+\mathcal{O}\left(|x|^{4}\right)\right) \\
\operatorname{coth} x & =\frac{1}{x}\left(1+\frac{x^{2}}{3}+\mathcal{O}\left(|x|^{4}\right)\right),
\end{aligned}
$$

noting in particular that $\cot x<1 / x$ for $x$ sufficiently small and positive, whereas $\operatorname{coth} x>1 / x$, so the inequalities are strict in one sense.

In order to study extension of the resulting metric to a smooth metric, or for later reference, just to a $C^{r}$ metric, we require the "cylinder to ball rule" in a slightly modified form. The proof is standard and we refer the reader to [4] for details.

Lemma 5.4 Consider a metric $g=\mathrm{d} r^{2}+b(r)^{2} \mathrm{~d} x^{2}$ defined and smooth on $0<r<r_{0}$ for some $r_{0}$, where $\mathrm{d} x^{2}$ is the metric for the circle $S^{1}$. Then $g$ extends to a $C^{2 l}(l \geq 1)$ metric across $r=0$ if and only if

$$
\lim _{r \rightarrow 0^{+}} b(r)=0, \quad \lim _{r \rightarrow 0^{+}} b^{\prime}(r)=1 \text { and } \lim _{r \rightarrow 0^{+}} \frac{\mathrm{d}^{2 k} b(r)}{\mathrm{d} r^{2 k}}=0(k=1, \ldots, l) .
$$

If further $\lim _{r \rightarrow 0^{+}} \frac{\mathrm{d}^{2(l+1)} b(r)}{\mathrm{d} r^{2(l+1)}} \neq 0$, then the extension is not $C^{2 l+1}$.

Theorem 5.5 For $A>0, k \neq 0$, the soliton metric corresponding to the orbit through the origin of the dynamical system (34) is not complete and cannot be extended to a complete metric. 
Proof Recall that $u=b^{\prime} / b, v=a^{\prime} / a$. Then $\xi \equiv 0$ implies that $A u+k v \equiv 0$, so that, on integrating, $b^{A} a^{k}=\alpha$ constant. Then $\eta=\frac{\sqrt{k^{2}+A^{2}}}{A} v=\frac{\sqrt{k^{2}+A^{2}}}{A} \frac{a^{\prime}}{a}$. Integration for $t \nearrow T$ now yields

$$
a(t)=(T-t)^{\frac{-c_{2} A}{\sqrt{k^{2}+A^{2}}}}+o(T-t),
$$

where $o(T-t)$ is interpreted as the condition $\lim _{t \nearrow T} \frac{o(T-t)}{T-t}=0$.

On the other hand $b=a^{-k / A}$ is given by

$$
b(t)=(T-t)^{\frac{c_{2} k}{\sqrt{k^{2}+A^{2}}}}+o(T-t) .
$$

By Corollary 5.3, $\left|\frac{c_{2} k}{\sqrt{k^{2}+A^{2}}}\right|<1$, so that the conditions for extension given by Lemma 5.4 are not satisfied

The next case is perhaps the most interesting in respect of the example it produces.

Case (iii) $A=0, k \neq 0$ The dynamical system (34) now takes the form

$$
\left\{\begin{array}{l}
\eta^{\prime}=\eta \rho+k \\
\rho^{\prime}=\eta^{2}
\end{array}\right.
$$

Then for $k \neq 0$ there are no fixed points and on replacing $\eta$ with $-\eta$, it is no loss of generality to suppose that $k>0$. Note also that given a solution $(\eta(t), \rho(t))$, then $\eta$ satisfies the ordinary differential equation:

$$
\eta \eta^{\prime \prime}-\left(\eta^{\prime}\right)^{2}+k \eta^{\prime}-\eta^{4}=0 .
$$

We study the orbit with initial condition $(\eta(0), \rho(0))=(0,0)$. The corresponding soliton will determine a soliton structure on a surface whose corresponding metric factors to the cylinder which we will show by the "cylinder to ball" rule, extends to a complete $C^{2}$ metric on the 2 -sphere $S^{2}$.

The derivatives

$$
\begin{aligned}
& (\eta+\rho)^{\prime}=\eta(\eta+\rho)+k \\
& (\eta-\rho)^{\prime}=-\eta(\eta-\rho)+k
\end{aligned}
$$

show that the orbit enters the slice $\eta+\rho>0, \eta-\rho>0$ and remains there. Since for $t>0$, we have $\eta(t)>\rho(t)>0$, then $\rho^{\prime}>\rho^{2}$ and comparison with the solution $1 /(\alpha-t)$ ( $\alpha$ constant) to the equation $\rho_{0}^{\prime}=\rho_{0}{ }^{2}$, as for the last case, shows that there exists a time $T<\infty$ such that $\lim _{t \nearrow T} \rho(t)=+\infty$. 
Lemma 5.6 For all $t$ such that $0<t<T, \rho(t)<\frac{1}{T-t}$ and $\eta(t)>\sqrt{k} \cot (\sqrt{k}(T-$ $t)$ ); furthermore, $T>\frac{\pi}{2 \sqrt{k}}$.

Proof The inequality $\frac{\mathrm{d} \rho}{\mathrm{d} t}>\rho^{2}$ implies that $\frac{\mathrm{d}}{\mathrm{d} t}\left(-\frac{1}{\rho}\right)>1$. Integration from $t$ to $T$ now shows that $\rho(t)<1 /(T-t)$, as required.

On the other hand, $\eta^{\prime}<\eta^{2}+k$ which implies that $\frac{\mathrm{d}}{\mathrm{d} t} \arctan \left(\frac{\eta}{\sqrt{k}}\right)<\sqrt{k}$. Integration from $t$ to $T$ now yields

$$
\frac{\pi}{2}-\arctan \left(\frac{\eta(t)}{\sqrt{k}}\right)<\sqrt{k}(T-t),
$$

from which we deduce the estimate on $\eta$. The estimate on $T$ is obtained by taking the limit $t \rightarrow 0^{+}$in the above.

We aim to show that

$$
\lim _{t \nearrow T}\left(\eta(t)-\frac{1}{T-t}\right)=0
$$

this is crucial to establish completeness of the extension of the induced metric that arises from this orbit. However, in order to proceed, we need more precise information on the relation between $T$ and the constant $k$.

Theorem 5.7 Let $(\eta(t), \rho(t))$ be the orbit of the dynamical system (38) with initial condition $(\eta(0), \rho(0))=(0,0)$. Then there exists a number $R>0$, independent of $k$, such that the bound $T=T(k)$ where $\lim _{t \nearrow T} \eta(t)=+\infty$, is given by

$$
T=\frac{R}{\sqrt{k}} \text {. }
$$

Remark 5.8 Experimentation with the program Maple (see Remark 5.11 below), indicates that the value of $R$ is approximately given by 1.9647 .

Proof As noted in Section 4, by analyticity of the first order system, the solution $(\eta(t), \rho(t))$ is analytic. Furthermore, for the solution passing through the origin, both $\eta(t)$ and $\rho(t)$ are odd functions. It follows therefore that for both functions, their limit as $t \searrow-T$ is $-\infty$. In particular, if we take an analytic development about $t=0$, then the radius of convergence for both functions must be $T$. 
Write

$$
\eta(t)=\sum_{l=0}^{\infty} \alpha_{l} t^{2 l+1}, \quad \rho(t)=\sum_{l=0}^{\infty} \beta_{l} t^{2 l+1},
$$

where $\alpha_{0}=k$ and $\beta_{0}=0$. The equations (38) give the following recurrence relations between the coefficients:

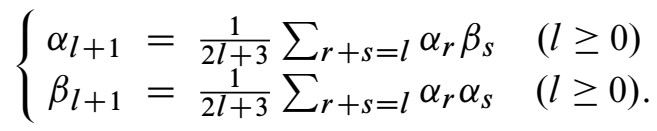

Note for what follows below, that $b_{1}=\frac{1}{3} k^{2}$. We claim that $\alpha_{l}=0$ for $l$ odd, $l \geq 1$ and that $\beta_{m}=0$ for $m$ even, $m \geq 0$. To see this, we apply induction. So suppose the claim is true for all $l, m \leq l_{0}$, for some positive integer $l_{0}$. If $l_{0}$ is even, then

$$
\alpha_{l_{0}+1}=\frac{1}{2 l_{0}+3} \sum_{r+s=l_{0}} \alpha_{r} \beta_{s},
$$

with $r+s$ even. So in each term on the RHS, either $r$ is even, in which case so is $s$ and by our induction hypothesis $\beta_{s}=0$, or $r$ is odd, in which case, once more by our induction hypothesis, $\alpha_{r}=0$, so that $\alpha_{l_{0}+1}=0$. On the other hand, if $l_{0}$ is odd, then

$$
\beta_{l_{0}+1}=\frac{1}{2 l_{0}+3} \sum_{r+s=l_{0}} \alpha_{r} \alpha_{s},
$$

with $r+s$ odd. So now in each term on the RHS, one of $r$ or $s$ must be odd and so the corresponding coefficient must vanish and $\beta_{l_{0}+1}=0$.

It therefore follows that $\eta(t)$ and $\rho(t)$ have expansions about $t=0$ of the form:

$$
\left\{\begin{array}{l}
\eta(t)=\sum_{l=0}^{\infty} a_{l} t^{4 l+1}, \quad\left(a_{0}=k\right) \\
\rho(t)=\sum_{m=0}^{\infty} b_{m} t^{4 m+3}, \quad\left(b_{0}=\frac{1}{3} k^{2}\right)
\end{array}\right.
$$

with corresponding recurrence relations:

$$
\left\{\begin{array}{lll}
a_{l+1}=\frac{1}{4 l+5} \sum_{r+s=l} a_{r} b_{s} & (l \geq 0), a_{0}=k \\
b_{l}=\frac{1}{4 l+3} \sum_{r+s=l} a_{r} a_{s} & (l \geq 0) .
\end{array}\right.
$$

The coefficients are now all strictly positive and we claim that $\lim _{n \rightarrow \infty} \frac{b_{n+1}}{b_{n}}=\frac{k^{2}}{R^{4}}$ for some number $R>0$, independent of $k$. By the estimates on $\rho$, we know that $\lim _{n \rightarrow \infty} \frac{b_{n+1}}{b_{n}}$ exists, is positive and finite and by the rule of d'Alembert, equals $T^{-4}$ (since the power of each successive term in the series increases by 4 ). 
We prove by induction that

$$
\begin{array}{ll}
a_{n}=k^{2 n+1} p(n) & (p(0)=1) \\
b_{n}=k^{2 n+2} q(n) & \left(q(0)=\frac{1}{3}\right)
\end{array}
$$

for numbers $p(n), q(n)$ independent of $k$. This is certainly true for $a_{0}$ and $b_{0}$, so suppose the property is true for all $a_{n}, b_{m}$ with $n, m \leq n_{0}$, for some positive integer $n_{0}$. Then

$$
a_{n_{0}+1}=\frac{1}{4 n_{0}+5} \sum_{r+s=n_{0}} a_{r} b_{s}
$$

where each term on the RHS has the form

$$
\begin{aligned}
a_{r} b_{s} & =k^{2 r+1} k^{2 s+2} p(r) q(s) \\
& =k^{2\left(n_{0}+1\right)+1} p(r) q(s),
\end{aligned}
$$

so that $a_{n_{0}+1}=k^{2\left(n_{0}+1\right)+1} p\left(n_{0}+1\right)$, where $p\left(n_{0}+1\right)=\frac{1}{4 n_{0}+5} \sum_{r+s=n_{0}} p(r) q(s)$.

On the other hand,

$$
b_{n_{0}+1}=\frac{1}{4 n_{0}+7} \sum_{r+s=n_{0}+1} a_{r} a_{s} .
$$

With the previous property established, that $a_{n_{0}+1}=k^{2\left(n_{0}+1\right)+1} p\left(n_{0}+1\right)$, we see that each term on the RHS has the form

$$
\begin{aligned}
a_{r} a_{s} & =k^{2 r+1} k^{2 s+1} p(r) p(s) \\
& =k^{2\left(n_{0}+1\right)+2} p(r) p(s),
\end{aligned}
$$

so that $b_{n_{0}+1}=k^{2\left(n_{0}+1\right)+2} q\left(n_{0}+1\right)$, where $q\left(n_{0}+1\right)=\frac{1}{4 n_{0}+7} \sum_{r+s=n_{0}+1} p(r) p(s)$. But now $\frac{b_{n+1}}{b_{n}}=k^{2} \frac{q(n+1)}{q(n)}$ so that

$$
\lim _{n \rightarrow \infty} \frac{b_{n+1}}{b_{n}}=k^{2} \lim _{n \rightarrow \infty} \frac{q(n+1)}{q(n)},
$$

and the result follows.

Write $e^{s}=1 /(T-t)$ and choose $k$ such that $T=1$. By Theorem 5.7, this is possible. Thus $t=0$ precisely when $s=0$. Our aim is to establish the following theorem.

Theorem 5.9 Let $x(s)=\eta\left(T-e^{-s}\right)-e^{s}$ and $y(s)=\rho\left(T-e^{-s}\right)-e^{s}$, where $\eta$ and $\rho$ satisfy the dynamical system (38), then $x(s), y(s) \rightarrow 0$ as $s \rightarrow \infty$. 
As a first step, we prove the following lemma.

Lemma 5.10 $\lim _{s \rightarrow \infty} e^{-s} \eta\left(T-e^{-s}\right)=\lim _{s \rightarrow \infty} e^{-s} \rho\left(T-e^{-s}\right)=1$.

Proof Set $\widetilde{\eta}(s)=e^{-s} \eta\left(T-e^{-s}\right), \widetilde{\rho}(s)=e^{-s} \rho\left(T-e^{-s}\right)$. Then (38) is equivalent to the non-autonomous system:

$$
\left\{\begin{array}{l}
\widetilde{\eta}(s)=-\widetilde{\eta}(s)+\widetilde{\eta}(s) \widetilde{\rho}(s)+k e^{-2 s} \\
\widetilde{\rho}(s)=-\widetilde{\rho}(s)+\widetilde{\eta}(s)^{2} .
\end{array}\right.
$$

Lemma 5.6 and the inequality $\eta\left(T-e^{-s}\right)>\rho\left(T-e^{-s}\right)>0$ for $s>0$, give the following information:

$$
\text { for } s>0, \quad \widetilde{\eta}(s)>\widetilde{\rho}(s)>0, \quad \widetilde{\rho}(s)<1 .
$$

We claim that $\lim _{s \rightarrow \infty}(\widetilde{\eta}-\widetilde{\rho})(s)=0$. Indeed,

$$
\begin{aligned}
(\widetilde{\eta}-\widetilde{\rho})^{\prime}(s) & =-(1+\widetilde{\eta})(\widetilde{\eta}-\widetilde{\rho})+k e^{-2 s} \\
& \leq-(\widetilde{\eta}-\widetilde{\rho})+k e^{-2 s} .
\end{aligned}
$$

Now comparison with the ODE: $\alpha^{\prime}(s)=-\alpha(s)+k e^{-2 s}$ with solution $\alpha(s)=B e^{-s}-$ $k e^{-2 s}$ ( $B$ constant), shows that $(\widetilde{\eta}-\widetilde{\rho})(s) \rightarrow 0$ as $s \rightarrow \infty$ (keeping in mind that $(\widetilde{\eta}-\widetilde{\rho})(s)>0$ for all $s>0)$.

From Lemma 5.6, $\eta(t)>\sqrt{k} \cot (\sqrt{k}(T-t))$, which implies that $\eta\left(T-e^{-s}\right)>e^{s}+$ $\mathcal{O}\left(e^{-s}\right)$, ie that $\widetilde{\eta}(s)>1+\mathcal{O}\left(e^{-2 s}\right)$. Since $\widetilde{\rho}(s)<1$, for all $s>0$ and $(\widetilde{\eta}-\widetilde{\rho})(s) \rightarrow 0$ as $s \rightarrow \infty$, it now follows that the $\operatorname{limits} \lim _{s \rightarrow \infty} \widetilde{\eta}(s)$ and $\lim _{s \rightarrow \infty} \widetilde{\rho}(s)$ both exist and are equal to 1 .

Remark 5.11 In fact, we can use this lemma to estimate the value of the radius of convergence $R$ of the series in Theorem 5.7 using Maple. In effect, the program plots the orbit through the origin for various values of $k$ and there is precisely one value of this constant for which it terminates at the point $(1,1)$. Since $T=1$, the value of $R$ is then given by $\sqrt{k}$.

Proof of Theorem 5.9 We refer the reader to Figure 3 for a plot of the solution. It is our aim to show that the point $x(s), y(s))$ converges asymptotically to the two lines $x-y=0$ and $2 x+y=0$, from which the result will follow.

The functions $x(s)$ and $y(s)$ satisfy the non-autonomous system

$$
\left\{\begin{array}{l}
x^{\prime}=e^{-s} x y+x+y+k e^{-s} \\
y^{\prime}=e^{-s} x^{2}+2 x
\end{array}\right.
$$




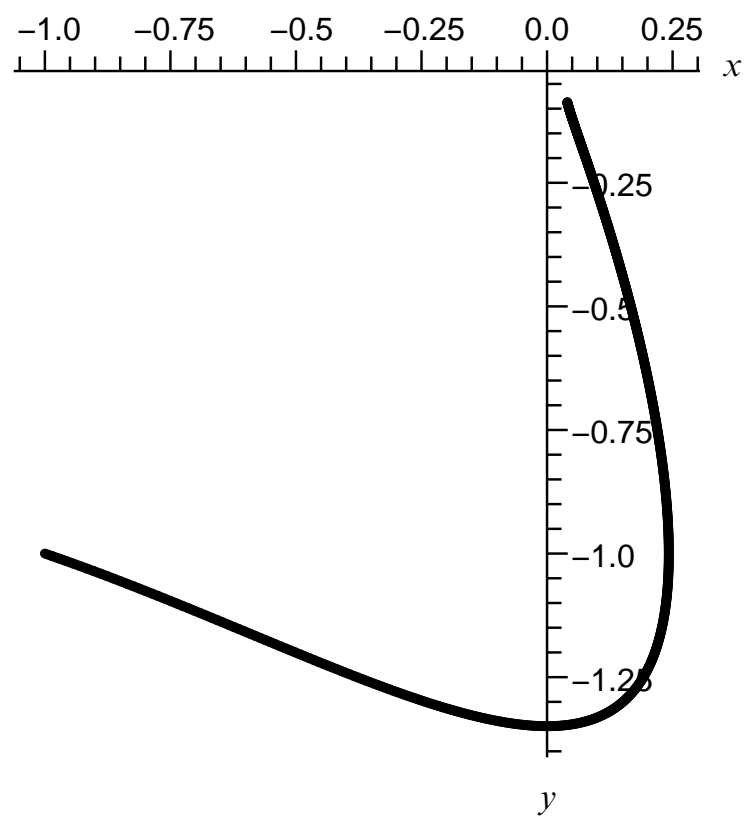

Figure 3: Trace of the solution curve to the non-autonomous dynamical system (40) with initial condition $x(0)=y(0)=-1$ and with $k$ taken to be 3.8602 .

We are interested in the orbit with initial condition $x(0)=y(0)=-1$ (since $\eta(0)=$ $\rho(0)=0)$. Then we have $y(s)<0, x(s)>y(s)$ for all $s>0$ and by Lemma 5.10:

$$
\lim _{s \rightarrow \infty} e^{-s} x(s)=\lim _{s \rightarrow \infty} e^{-s} y(s)=0 .
$$

For $s>0$, write $x(s)=y(s)+\mu(s)$, where $\mu(s)>0$. Then

$$
\mu^{\prime}=x^{\prime}-y^{\prime}=-\mu\left(1+x e^{-s}\right)+k e^{-s} .
$$

We claim that $\lim _{s \rightarrow \infty} \mu(s)=0$. Now the ODE:

$$
\widetilde{\mu}^{\prime}=-\widetilde{\mu}(1+\delta)+k e^{-s} \quad(\delta \neq 0)
$$

has general solution $\widetilde{\mu}=C e^{-(1+\delta) s}+\frac{k}{\delta} e^{-s}$. We now compare $\mu$ with $\widetilde{\mu}$; so for a given $0<\varepsilon<1$, let $s_{0}>0$ be such that $\left|x(s) e^{-s}\right|<\varepsilon$ for all $s \geq s_{0}$ and consider the 
two ODEs:

$$
\begin{aligned}
& \mu_{0}^{\prime}=-\mu_{0}(1+\varepsilon)+k e^{-s} \\
& \mu_{1}^{\prime}=-\mu_{1}(1-\varepsilon)+k e^{-s}
\end{aligned}
$$

with initial conditions $\mu_{0}\left(s_{0}\right)=\mu_{1}\left(s_{0}\right)=\mu\left(s_{0}\right)$. Then since $\mu_{1}^{\prime}>\mu^{\prime}>\mu_{0}^{\prime}$ and $\lim _{s \rightarrow \infty} \mu_{1}(s)=\lim _{s \rightarrow \infty} \mu_{0}(s)=0$, we conclude that $\lim _{s \rightarrow \infty} \mu(s)=0$.

Now define $v(s)=2 x(s)+y(s)$. Then

$$
v^{\prime}=v\left(2+e^{-s} x\right)-\mu e^{-s} x .
$$

Note that $\lim _{s \rightarrow \infty} e^{-s} v(s)=0$. Firstly, observe that $v(s)<0$ for all $s>0$, since initially $v(0)<0$ and if $v(s)=0$ for some $s>0$, then let $s_{0}$ be the first such time. Then

$$
v^{\prime}\left(s_{0}\right)=-\mu\left(s_{0}\right) e^{-s_{0}} x\left(s_{0}\right) .
$$

But since $y\left(s_{0}\right)<0$, then $x\left(s_{0}\right)>0$ (since $\left.v\left(s_{0}\right)=2 x\left(s_{0}\right)+y\left(s_{0}\right)=0\right)$, which implies that $\nu^{\prime}\left(s_{0}\right)<0$, a contradiction.

For $0<\varepsilon<1$, let $s_{0}>0$ be such that $\left|e^{-s} x(s)\right|, \mu(s)\left|e^{-s} x(s)\right|<\varepsilon$ for $s>s_{0}$. Then for $s>s_{0}$.

$$
\begin{aligned}
v^{\prime} & \leq v\left(2-\left|e^{-s} x\right|\right)-\mu e^{-s} x \\
& <v(2-\varepsilon)+\varepsilon .
\end{aligned}
$$

We now compare $v$ with the solution to the ODE

$$
\nu_{1}^{\prime}=v_{1}(2-\varepsilon)+\varepsilon
$$

with initial condition $v_{1}\left(s_{0}\right)=v\left(s_{0}\right)$. Specifically, the solution is given by

$$
v_{1}(s)=C_{1} e^{(2-\varepsilon) s}-\frac{\varepsilon}{2-\varepsilon} \text { with } C_{1}=\left(v\left(s_{0}\right)+\frac{\varepsilon}{2-\varepsilon}\right) e^{-(2-\varepsilon) s_{0}} .
$$

Then by comparison, for $s>s_{0}$,

$$
\nu(s)<C_{1} e^{(2-\varepsilon) s}-\frac{\varepsilon}{2-\varepsilon},
$$

which, on multiplying through by $e^{-s}$ gives

$$
\nu(s) e^{-s}<C_{1} e^{(1-\varepsilon) s}-\frac{\varepsilon e^{-s}}{2-\varepsilon} .
$$

Now take the limit as $s \rightarrow \infty$. Then if $C_{1}<0$, the right-hand side tends to $-\infty$, which contradicts the fact that $v(s) e^{-s} \rightarrow 0$. Hence $C_{1} \geq 0$. But our choice of $\varepsilon$ is arbitrary within the interval $(0,1)$ and since $v(s)<0$, we conclude from $(41)$ that $\lim _{s \rightarrow \infty} v(s)=0$. This completes the proof of the Theorem. 
Recall that the corresponding soliton metric has the form $g=\mathrm{d} t^{2}+a(t)^{2}\left(\mathrm{~d} x^{2}\right)^{2}+$ $b(t)^{2}\left(\mathrm{~d} x^{2}\right)^{2}$, where, since $A=0$, we have $v(t) \equiv 0$ and $a(t)$ is constant. Furthermore, $\eta(t)=-u(t)=-b^{\prime}(t) / b(t)$.

Write $r=T-t$, so that $b^{\prime}(t)=-b^{\prime}(r)$ and $\eta(r)=b^{\prime}(r) / b(r)$, and $t \nearrow T$ if and only if $r \rightarrow 0^{+}$. Let us now apply the "cylinder to ball rule" as expressed by Lemma 5.4, to the metric $\mathrm{d} r^{2}+b(r)^{2}\left(\mathrm{~d} x^{3}\right)^{2}$.

First, on recalling that $e^{s}=1 / r$, from Theorem 5.9, we have

$$
\eta(r)=\frac{1}{r}+x(r), \quad \text { where } x(r) \rightarrow 0 \text { as } r \rightarrow 0^{+} .
$$

Thus, for $r, r_{0}>0$, we have

$$
b(r)=B \exp \left(\int_{r_{0}}^{r} \eta(\zeta) \mathrm{d} \zeta\right)=B\left(\frac{r}{r_{0}}\right) \exp \int_{r_{0}}^{r} x(\zeta) \mathrm{d} \zeta
$$

for some constant $B$, which, since $\lim _{r \rightarrow 0^{+}} x(r)=0$, shows that $\lim _{r \rightarrow 0^{+}} b(r)=0$. Furthermore the constants $r_{0}$ and $B$ can be chosen so that $\lim _{s \rightarrow 0^{+}} b^{\prime}(s)=1$. We shall choose these more selectively below.

From (42), we have

$$
b^{\prime \prime}(r)=\frac{2 B}{r_{0}} x(r)+\frac{B r}{r_{0}} x^{\prime}(r) .
$$

But then $x^{\prime}(r)=-x^{\prime}(s) / r$, so that

$$
b^{\prime \prime}(r)=\frac{2 B}{r_{0}} x(r)-\frac{B}{r_{0}} x^{\prime}(s) .
$$

It therefore follows that $\lim _{r \rightarrow 0^{+}} b^{\prime \prime}(r)=0$ if and only if $\lim _{s \rightarrow \infty} x^{\prime}(s)=0$. But the latter affirmation is a consequence of Theorem 5.9 and the expression for $x^{\prime}(s)$ given by (40). It now follows that the extension of the metric to $r=0$ is of class $C^{2}$.

We now want to combine the two ends $t \nearrow T$ and $t \searrow-T$, alternatively, $r \rightarrow 0^{+}$and $r \rightarrow 2 T^{-}$. Recall that $\eta(t)$ is an odd function of $t$. Write

$$
\begin{aligned}
b(r) & =B \exp \left(\int_{r_{0}}^{r} \eta(\zeta) \mathrm{d} \zeta\right) \\
& =B \exp \left\{\int_{r_{0}}^{r}\left(\frac{1}{\zeta}-\frac{1}{2 T-\zeta}+\left(\eta(\zeta)-\frac{1}{\zeta}+\frac{1}{2 T-\zeta}\right)\right) \mathrm{d} \zeta\right\}
\end{aligned}
$$


Then we have by analogy that $\eta(r)+\frac{1}{2 T-r} \rightarrow 0$ as $r \rightarrow 2 T^{-}$and clearly

$$
\int_{r_{0}}^{r}\left(\eta(\zeta)-\frac{1}{\zeta}+\frac{1}{2 T-\zeta}\right) d \zeta
$$

is bounded for all $r_{0}, r \in(0,2 T)$. Note also that the integrand $\eta(\zeta)-\frac{1}{\zeta}+\frac{1}{2 T-\zeta}$ is odd with respect to symmetry about the point $\zeta=T$; that is, with respect to the reflection $\zeta \mapsto 2 T-\zeta$. In particular, if we set $r_{0}=T$, then the function

$$
f(r)=\exp \left\{\int_{T}^{r}\left(\eta(\zeta)-\frac{1}{\zeta}+\frac{1}{2 T-\zeta}\right) \mathrm{d} \zeta\right\}
$$

has the property that $f(r)=f(2 T-r)$.

Let $L=\lim _{r \rightarrow 0^{+}} f(r)=\lim _{r \rightarrow 2 T^{-}} f(r)$. Then $b(r)=B \frac{r(2 T-r)}{T^{2}} f(r)$ and $\lim _{r \rightarrow 0^{+}} b^{\prime}(r)=$ $\lim _{r \rightarrow 2 T^{-}} b^{\prime}(r)=\frac{2 B L}{T}$. So, on choosing $B=T / 2 L$, we are now guaranteed that the metric $g$ extends to both ends $r=0,2 T$ in a $C^{2}$ way.

Let us now assemble the key aspects of this example. In the first instance, the soliton metric $g$ is defined on the manifold $0<r<2 T, x^{2}, x^{3} \in \mathbf{R}$. The expression (32) for the scalar curvature, shows that it does not have constant curvature. The corresponding soliton flow is given by Theorem 3.1, that is by the vector field:

$$
E=(\eta-\rho) \frac{\partial}{\partial r}-k x^{3} \frac{\partial}{\partial x^{3}} .
$$

Since $A=0$, the soliton structure constructed above can be considered as corresponding to one on the surface diffeomorphic to $\mathbf{R}^{2}$ given by $\left\{\left(r, x^{3}\right): 0<r<2 T, x^{3} \in \mathbf{R}\right\}$. Since $k+A=k$ is non-zero and $b^{\prime}(s) \neq 0$, by Theorem 3.1, it is not of gradient type. The metric $g$ factors by the action of $2 \pi \mathbf{Z}$ on $\mathbf{R}$, to give a metric on $(0,2 T) \times S^{1}$, but clearly the soliton flow given by (43) doesn't. Furthermore, by the cylinder to ball rule, $g$ extends across $r=0,2 T$ to a $C^{2}$ metric on the sphere $S^{2}$. Notice also, that by our estimates on the asymptotic behaviour of $\eta$ and $\rho$, the coefficient $\eta-\rho$ of $\partial / \partial r$ in $E$ has removable singularities at $r=0,2 T$ and the limits $\lim _{r \rightarrow 0^{+}}(\eta(r)-\rho(r))$ and $\lim _{r \rightarrow 2 T^{-}}(\eta(r)-\rho(r))$ both vanish. Finally, as we saw in the discussion following Theorem 3.1, the absolute value of the constant $k$ is only defined up to homothety, so we may as well choose it so that the value of $T=T(k)$ is 1 . We summarise all this with the following theorem.

Theorem 5.12 The orbit through the origin of the dynamical system (38) determines a steady soliton metric $g$ of non-constant curvature on the surface $(0,2) \times \mathbf{R}$. The 
soliton is of non-gradient type and its flow is given by the vector field (43). The metric $g$ factors to a metric on the cylinder $(0,2) \times S^{1}$ and then by the cylinder to ball rule, extends to a $C^{2}$ metric on the sphere $S^{2}$.

Acknowledgement The author thanks the referee for carefully reading through the manuscript and in particular for pointing out a mistake in one of the examples. This work was carried out under the award of a Délégation auprès du Centre National de la Recherche Scientifique.

\section{References}

[1] P Baird, L Danielo, Three-dimensional Ricci solitons which project to surfaces, J. Reine Angew. Math. 608 (2007) 65-91 MR2339469

[2] R L Bryant, Ricci flow solitons in dimension three with $\mathrm{SO}(3)$-symmetries, preprint, Duke Univ. (2005)

[3] B Chow, S-C Chu, D Glickenstein, C Guenther, J Isenberg, T Ivey, D Knopf, P Lu, F Luo, L Ni, The Ricci flow: techniques and applications., Mathematical Surveys and Monographs 135, Amer. Math. Soc. (2007) MR2302600 Part I: Geometric aspects

[4] B Chow, D Knopf, The Ricci flow: an introduction, Mathematical Surveys and Monographs 110, Amer. Math. Soc. (2004) MR2061425

[5] J Dieudonné, Foundations of modern analysis, Academic Press, New York (1969) MR0349288

[6] C Guenther, J Isenberg, D Knopf, Stability of the Ricci flow at Ricci-flat metrics, Comm. Anal. Geom. 10 (2002) 741-777 MR1925501

[7] C Guenther, J Isenberg, D Knopf, Stability of Ricci nilsolitons, preprint (2006)

[8] R S Hamilton, The Ricci flow on surfaces, from: "Mathematics and general relativity (Santa Cruz, CA, 1986)”, Contemp. Math. 71, Amer. Math. Soc. (1988) 237-262 MR954419

[9] T Ivey, Ricci solitons on compact three-manifolds, Differential Geom. Appl. 3 (1993) 301-307 MR1249376

[10] J Lott, On the long-time behavior of type-III Ricci flow solutions, Math. Ann. 339 (2007) 627-666 MR2336062

[11] G Perelman, The entropy formula for the Ricci flow and its geometric applications arXiv:math.DG/0211159

[12] N Sesum, Linear and dynamical stability of Ricci flat metrics, preprint, Courant Institute 
Département de Mathématiques, Université de Bretagne Occidentale 6 av. Victor Le Gorgeu - CS 93837, 29238 Brest Cedex, France

Paul.Baird@univ-brest.fr

Proposed: Tobias Colding

Seconded: Ben Chow, Gang Tian
Received: 27 August 2008

Revised: 17 December 2008 